\title{
Experimental bounds on sterile neutrino mixing angles
}

\author{
Oleg Ruchayskiy ${ }^{a}$ and Artem Ivashko ${ }^{b, c}$ \\ ${ }^{a}$ CERN Physics Department, Theory Division, \\ CH-1211 Geneva 23, Switzerland \\ ${ }^{b}$ Instituut-Lorentz for Theoretical Physics, Universiteit Leiden, \\ Niels Bohrweg 2, Leiden, The Netherlands \\ ${ }^{c}$ Department of Physics, Kiev National Taras Shevchenko University, \\ Glushkov str. 2 building 6, Kiev, 03022, Ukraine \\ E-mail: oleg.ruchayskiy@cern.ch, ivashko@lorentz.leidenuniv.nl
}

Abstract: We derive bounds on the mixing between the Standard Model ("active") neutrinos and their right-chiral ("sterile") counterparts in the see-saw models, by combining neutrino oscillation data and results of direct experimental searches. We demonstrate that the mixing of sterile neutrinos with any active flavour can be significantly suppressed for the values of the angle $\theta_{13}$ measured recently by Daya Bay and RENO experiments. We reinterpret the results of searches for sterile neutrinos by the PS191 and CHARM experiments, considering not only charged current but also neutral current-mediated decays, as applicable in the case of see-saw models. The resulting lower bounds on sterile neutrino lifetime are up to an order of magnitude stronger than previously discussed in the literature. Combination of these results with the upper bound on the lifetime coming from primordial nucleosynthesis rule out the possibility that two sterile neutrinos with the masses between $10 \mathrm{MeV}$ and the pion mass are solely responsible for neutrino flavour oscillations. We discuss the implications of our results for the Neutrino Minimal Standard Model (the $\nu$ MSM).

Keywords: Beyond Standard Model, Rare Decays, Neutrino Physics

ARXiv EPRINT: 1112.3319 


\section{Contents}

1 Introduction 1

2 Sterile neutrino Lagrangian 3

3 Solution of the see-saw equations 4

3.1 Parametrization of the Dirac mass matrix 4

3.2 Normal hierarchy 5

$\begin{array}{lll}3.3 & \text { Inverted hierarchy } & 7\end{array}$

3.4 Minimal mixing angles in the $\nu$ MSM $\quad 9$

4 Experimental bounds on sterile neutrino mixings $\quad 11$

$\begin{array}{lll}4.1 \text { Peak searches } & 11\end{array}$

$\begin{array}{lll}4.2 & \text { Fixed target experiments and neutral currents contribution } & 12\end{array}$

\begin{tabular}{ll}
4.3 & Reinterpretation of the PS191 and CHARM experiments \\
\hline
\end{tabular}

$\begin{array}{ll}4.4 \text { A note on Majorana vs Dirac neutrinos } & 15\end{array}$

$\begin{array}{lll}5 & \text { Results } & \mathbf{1 5}\end{array}$

$\begin{array}{lll}5.1 \text { Bounds on the mixing angles of sterile neutrinos } & 16\end{array}$

$\begin{array}{ll}5.2 & \text { The lower bound on the lifetime of sterile neutrinos } \\ \end{array}$

$\begin{array}{llr}6 & \text { Discussion } & 17\end{array}$

$\begin{array}{lr}\text { A Sterile neutrino lifetime } & 20\end{array}$

$\begin{array}{ll}\text { B PMNS parametrization } & 21\end{array}$

C Ratio of sterile neutrino mixing angles for $|z| \sim 1 \quad 21$

\section{Introduction}

Transitions between neutrinos of different flavours (see e.g. [1] for a review and refs. [2-4] for the recent update of experimental values) are among the few firmly established phenomena beyond the Standard Model of elementary particles. The simplest explanation is provided by the "neutrino flavour oscillations" - non-diagonal matrix of neutrino propagation eigenstates in the weak charge basis. While the absolute scale of neutrino masses is not established, particle physics measurements put the sum of their masses below $2 \mathrm{eV}$ [5] while from the cosmological data one can infer an upper bound of $0.58 \mathrm{eV}$ at $95 \% \mathrm{CL}$ [6].

A traditional explanation of the smallness of neutrino masses is provided by the seesaw mechanism [7-10]. It assumes the existence of several right-handed neutrinos, coupled 
to their Standard Model (SM) counterparts via the Yukawa interaction, providing the Dirac masses, $M_{\mathrm{D}}$, for neutrinos. The Yukawa interaction terms dictate the SM charges of the right-handed particles: they turn out to carry no electric, weak and strong charges; therefore they are often termed "singlet," or "sterile" fermions. Sterile neutrinos can thus have Majorana masses, $M_{s}$, consistent with the gauge symmetries of the Standard Model. If the Majorana masses are much larger than the Dirac ones, the type I seesaw formula [7-10] holds, expressing the mass matrix of observed neutrinos $\left(\mathcal{M}_{\nu}\right)$ via

$$
\mathcal{M}_{\nu}=-M_{\mathrm{D}} M_{s}^{-1} M_{\mathrm{D}}^{T},
$$

where $\mathcal{M}_{\nu}$ is a $3 \times 3$ matrix of active neutrino masses, mixings, and (possible) CP-violating phases. The masses of sterile neutrinos are given by the eigenvalues of their Majorana mass matrix (with the corrections of the order $M_{\mathrm{D}}^{2} / M_{s}^{2}$ ). They are much heavier than the active neutrino masses as a consequence of (1.1).

Numerous searches for sterile neutrinos in the mass range up to $\sim 100 \mathrm{GeV}$ had been performed (see the corresponding section in Particle Data Group [5], ${ }^{1}$ see also [11] and refs. therein). These searches provided upper bounds on the strength of interaction of these neutral leptons with the SM neutrinos of different flavours - active-sterile neutrino mixing angles $\vartheta_{\alpha}^{2} \propto\left|\frac{M_{\mathrm{D}, \alpha}}{M_{s}}\right|^{2}$ for sterile neutrino with the mass $M_{s}{ }^{2}$. These bounds then can be interpreted as lower bounds on the lifetime of sterile neutrinos $\tau_{s}$ via

$$
\tau_{s}^{-1}=\frac{G_{F}^{2} M_{s}^{5}}{96 \pi^{3}} \sum_{X} \vartheta_{\alpha}^{2} B_{X}^{(\alpha)}
$$

where the sum runs over various particles to which sterile neutrino can decay, depending on their mass $\left(\nu, e^{ \pm}, \mu^{ \pm}, \tau^{ \pm}, \pi, K\right.$, heavier mesons and baryons) and dimensionless quantities $B_{X}^{\alpha}$ depend on the branching ratios (see appendix A for details). The lower bound on the lifetime $\tau_{s}$ is usually dominated by the least constrained mixing angle, $\vartheta_{\tau}^{2}$ (as will be shown later).

This bound can be made stronger if one assumes that the same particles are also responsible for the neutrino oscillations. The see-saw formula (1.1) limits (at least partially) possible values of ratios of the mixing angles $\vartheta_{\alpha}^{2} / \vartheta_{\beta}^{2}$. In the simplest case when only two sterile neutrinos are present (the minimal number, required to explain two observed neutrino mass differences) the ratios of mixing angles varies within a limited range, see e.g. $[12,13]$. While this range can be several orders of magnitude large (owing to our ignorance of certain oscillation parameters, such as e.g. a CP-violating phase $[12,13])$, the implied (lower) bounds on the lifetime become much stronger, essentially being determined by the strongest, rather than the weakest direct bound on $\vartheta_{\alpha}$. When confronted with the upper bound from Big Bang Nucleosynthesis [14, 15], they seem to close the window of parameters for sterile neutrinos with the mass lighter than about $150 \mathrm{MeV}[12,16]$. It was

\footnotetext{
${ }^{1}$ http://pdglive. lbl.gov/Rsummary . brl?nodein=S077\&inscript=Y.

${ }^{2}$ Here and below we use the letter $\vartheta$ for active-sterile mixing angles (defined by eq. (3.7) below) while reserving $\theta_{12}, \theta_{13}$ and $\theta_{23}$ for the measured parameters of the active neutrinos matrix $\mathcal{M}_{\nu}$. These quantities $\vartheta_{\alpha}$ are often denoted $\left|V_{4 \alpha}\right|^{2}$ or $\left|U_{x \alpha}\right|^{2}$ in the experimental papers, to which we refer. Here and below the Greek letters $\alpha, \beta$ are flavour index $e, \mu, \tau$ and $i, j=1,2,3$ denote active neutrino mass eigenstates.
} 
argued in [17], however, that in the case of normal hierarchy there can be a small allowed window of parameters of sterile neutrinos with the mass below the pion mass.

In this paper we reanalyze restrictions on sterile neutrino lifetime in view of the recent results of the Daya Bay [18] and RENO [19] collaborations, that measured a non-zero mixing angle $\theta_{13}$ (see also $[20,21]$ ). We demonstrate that in the case when there are only two sterile neutrinos, responsible for the observed neutrino oscillations, the oscillation data allow for such a choice of the active-sterile Yukawa couplings that the mixing of sterile neutrinos with any given flavour can be strongly suppressed. This happens only for a non-zero values of $\theta_{13}$, in the range consistent with the current measurements $[2,4,18,19]$. We also confront our results with the recently reanalyzed bounds from primordial nucleosynthesis [22] and show that the window in the parameter space of sterile neutrinos with masses $10 \mathrm{MeV} \lesssim M_{s} \lesssim 140 \mathrm{MeV}$ discussed in previous works [17] (see also [12]) is closed. For larger masses the window remains open. The results of this paper partially overlap with [17] (also [23]), and we compare in the corresponding places to the previous works.

The paper is organized as follows: in section 2 we briefly describe the type I seesaw model we use. We then investigate the relations between different mixing angles imposed by the see-saw mechanism and demonstrate that the mixing with any flavour $\vartheta_{\alpha}^{2}$ can become suppressed (section 3). Section 4 is devoted to the overview of the experiments, searching for sterile neutrinos with the masses below $2 \mathrm{GeV}$, and the way one should interpret their results to apply to the see-saw models that we study. Section 5 summarizes our revised bounds on mixing angles and translates them into the resulting constraints on sterile neutrino lifetime (figures 7). We conclude in section 6, discussing implications of our results and confronting them with the bounds from primordial nucleosynthesis.

\section{Sterile neutrino Lagrangian}

The minimal way to add sterile neutrinos to the Standard Model is provided by the Type I see-saw model [7-10] (see also [24-27] and refs. therein):

$$
\Delta \mathcal{L}_{N}=\sum_{I, J=1}^{\mathcal{N}} i \bar{N}_{I} \partial_{\mu} \gamma^{\mu} N_{I}-\left(F_{\alpha I} \bar{L}_{\alpha} N_{I} \tilde{\Phi}-\frac{\left(M_{s}\right)_{I J}}{2} \bar{N}_{I}^{c} N_{J}+\text { h.c. }\right)
$$

where $F_{\alpha I}$ are new Yukawa couplings, $\Phi_{i}$ is the SM Higgs doublet, $\tilde{\Phi}_{i}=\epsilon_{i j} \Phi_{j}^{\dagger}$. This model is renormalizable, has the same gauge symmetries as the Standard Model, and contains $\mathcal{N}$ additional Weyl fermions $N_{I}$ - sterile neutrinos ( $N_{I}^{c}$ being the charge-conjugate fermion, in the chiral representation of Dirac $\gamma$-matrices $\left.N_{I}^{c}=i \gamma^{2} N_{I}^{\dagger}\right)$. The number of these singlet fermions must be $\mathcal{N} \geq 2$ to explain the data on neutrino oscillations. In the case of $\mathcal{N}=2$ there are 11 new parameters in the Lagrangian (2.1), while the neutrino mass/mixing matrix $\mathcal{M}_{\nu}$ has 7 parameters in this case. The situation is even more relaxed for $\mathcal{N}>2$. The see-saw formula (1.1) does not allow to fix the scale of Majorana and Dirac $M_{\mathrm{D}, \alpha I}=F_{\alpha I}\langle\Phi\rangle$ masses. In this work we will mostly concentrate on sterile neutrinos with their masses $M_{s}$ in the $\mathrm{MeV}-\mathrm{GeV}$ range - the range that is probed by the direct searches. To further simplify our analysis we will concentrate on the case when the masses 
of both sterile neutrinos are close to each other (so that $\Delta M \ll M_{s}$ ). One important example of such model is provided by the Neutrino Extended Standard Model (the $\nu \mathrm{MSM}$ ) $([28,29]$, see [16] for review). The $\nu$ MSM model contains 3 sterile neutrinos, whose masses are roughly of the order of those of other leptons in the Standard Model. Two of these particles (approximately degenerate in their mass) are responsible for baryogenesis and neutrino oscillations and the third one is playing the role of dark matter. The requirement of dark matter stability on cosmological timescales makes its coupling with the Standard Model species so feeble, that it does not contribute significantly to the neutrino oscillation pattern [30]. Therefore, when analyzing neutrino oscillations, the $N_{1}$ can be omitted from the Lagrangian and index $I$ in the sums runs through 2 and 3 only:

$$
\mathcal{L}_{\text {see-saw }}=\mathcal{L}_{S M}+\bar{N}_{I} i \partial_{\mu} \gamma^{\mu} N_{I}-M_{\mathrm{D}, \alpha I} \bar{\nu}_{\alpha} N_{I}-M_{\mathrm{D}, \alpha I}^{*} \bar{N}_{I} \nu_{\alpha}-M_{s} \bar{N}_{2}^{c} N_{3}-M_{s} \bar{N}_{3} N_{2}^{c}
$$

This parametrization coincides with [13, eq. (2.1)]. Note that we use basis of singlet neutrinos where the mass matrix is off-diagonal. Recent computation of the baryon asymmetry of the Universe in the $\nu \mathrm{MSM}$ [31] demonstrated that sterile neutrinos with the mass as low as several $\mathrm{MeV}$ can be responsible for baryogenesis and neutrino oscillations within the $\nu \mathrm{MSM}$. This prompts us to re-analyze the implication of negative direct experimental searches for the Yukawa couplings of sterile neutrinos with the $\mathrm{MeV}$ masses. We limit our analysis by $M_{s} \leq 2 \mathrm{GeV}$, as for the higher masses the existing experimental bounds do not probe the region of mixing angles, required to produce successful baryogenesis in the $\nu \mathrm{MSM}$ [31].

\section{Solution of the see-saw equations}

In this section we investigate how mixing angles between active and sterile neutrinos are related to parameters of the observable neutrino matrix $\mathcal{M}_{\nu}$. We will demonstrate that the mixing angle $\vartheta_{e}^{2}$ in the case of normal hierarchy and the mixing angles $\vartheta_{\mu}^{2}$ or $\vartheta_{\tau}^{2}$ in the case of inverted hierarchy, can become suppressed as we vary the parameters of the neutrino matrix away from their best-fit values (but within the experimentally allowed $3 \sigma$ bounds).

\subsection{Parametrization of the Dirac mass matrix}

We use the Pontecorvo-Maki-Nakagawa-Sakata (PMNS) parametrization of the neutrino matrix $\mathcal{M}_{\nu}$ (see e.g. eqs. (2.10) and (2.12) of [1])

$$
\mathcal{M}_{\nu}=V^{*} \operatorname{diag}\left(m_{1} e^{-2 i \zeta}, m_{2} e^{-2 i \xi}, m_{3}\right) V^{\dagger}
$$

where $V$ is the unitary matrix, whose explicit form is reminded in appendix B. Redefining a Dirac mass matrix as ${ }^{3}$

$$
M_{\mathrm{D}} \rightarrow \tilde{M}_{\mathrm{D}} \equiv V^{T} M_{\mathrm{D}},
$$

we can rewrite the see-saw relation (1.1) in the following form:

$$
\operatorname{diag}\left(m_{1} e^{-2 i \zeta}, m_{2} e^{-2 i \xi}, m_{3}\right)_{i j}=-\frac{\tilde{M}_{\mathrm{D}, i 2} \tilde{M}_{\mathrm{D}, j 3}+\tilde{M}_{\mathrm{D}, i 3} \tilde{M}_{\mathrm{D}, j 2}}{M_{s}},
$$

\footnotetext{
${ }^{3}$ The Dirac matrix $\tilde{M}_{\mathrm{D}}$ has indexes $I=2,3$ and $i, j=1,2,3$ - neutrino propagation basis.
} 


\begin{tabular}{|ll|ll|}
\hline \multicolumn{2}{|c|}{ Normal hierarchy } & \multicolumn{2}{c|}{ Inverted hierarchy } \\
\hline$\Delta m_{21}^{2}$ & $(7.09-8.19) \times 10^{-5} \mathrm{eV}^{2}$ & & \\
$\Delta m_{31}^{2}$ & $(2.14-2.76) \times 10^{-3} \mathrm{eV}^{2}$ & $\Delta m_{13}^{2}$ & $(2.13-2.67) \times 10^{-3} \mathrm{eV}^{2}$ \\
$\sin ^{2} \theta_{12}$ & $0.27-0.36$ & & \\
$\sin ^{2} \theta_{23}$ & $0.39-0.64$ & & \\
$\sin ^{2} \theta_{13}$ & $0.010-0.038(0.013-0.040)$ & & \\
\hline
\end{tabular}

Table 1. The $3 \sigma$ bounds on the parameters of the mass matrix $\mathcal{M}_{\nu}$, adopted from $[3,4,18,19]$. Here $\Delta m_{i j}^{2}=m_{i}^{2}-m_{j}^{2}$. The boundaries for inverted hierarchy are the same as for the normal one, unless written explicitly. The range of $\sin ^{2} \theta_{13}$ is taken from the data of the Daya Bay experiment [18] (the values in parentheses - from RENO [19]).

The rank of the active neutrino mass matrix $\mathcal{M}_{\nu}$ is 2 in the case of two sterile neutrinos, meaning that one of the masses $m_{i}$ is zero. Two choices of "hierarchies" of the mass eigenvalues are possible. The first one is called normal hierarchy $(\mathrm{NH})$ and corresponds to $0 \leq m_{1}<m_{2}<m_{3}$. The second one is called inverted hierarchy (IH) and is realized for $0 \leq m_{3}<m_{1}<m_{2}$.

Once the mass $M_{s}$ is fixed, the solutions of eq. (3.3) contain one unknown complex parameter, $z$. Its presence reflects a symmetry of the see-saw relation (3.3) under the change $\left(\tilde{M}_{\mathrm{D}, i 2}, \tilde{M}_{\mathrm{D}, i 3}\right) \rightarrow\left(z \tilde{M}_{\mathrm{D}, i 2}, z^{-1} \tilde{M}_{\mathrm{D}, i 3}\right)[32]$. It is this freedom that does not allow to fix the absolute scale of $\tilde{M}_{\mathrm{D}}$ (i.e. the value of $\vartheta^{2}$ ) even if $M_{s}$ is chosen.

The change $z \rightarrow z^{-1}$ is equivalent to the redefinition of $N_{2} \rightarrow N_{3}, N_{3} \rightarrow N_{2}$ together with shift of the Majorana phase $\xi \rightarrow \xi+\pi$ in (3.3). Therefore in subsequent analysis we will choose $|z| \geq 1$ without the loss of generality.

\subsection{Normal hierarchy}

For normal hierarchy the explicit see-saw relation is

$$
\operatorname{diag}\left(0, m_{2} e^{-2 i \xi}, m_{3}\right)_{i j}=-\frac{\tilde{M}_{\mathrm{D}, i 2} \tilde{M}_{\mathrm{D}, j 3}+\tilde{M}_{\mathrm{D}, i 3} \tilde{M}_{\mathrm{D}, j 2}}{M_{s}}
$$

Diagonal components of this matrix equation give

$$
\tilde{M}_{\mathrm{D}, 12} \tilde{M}_{\mathrm{D}, 13}=0, \quad \tilde{M}_{\mathrm{D}, 22} \tilde{M}_{\mathrm{D}, 23}=\frac{1}{2} m_{2} M_{s} e^{-2 i \xi}, \quad \tilde{M}_{\mathrm{D}, 32} \tilde{M}_{\mathrm{D}, 33}=\frac{1}{2} m_{3} M_{s}
$$

Using $m_{2}, m_{3} \neq 0$ we find that $\tilde{M}_{\mathrm{D}, 22}, \tilde{M}_{\mathrm{D}, 23}, \tilde{M}_{\mathrm{D}, 32}, \tilde{M}_{\mathrm{D}, 33}$ are all non-zero. Analysis of non-diagonal terms reveals that both $\tilde{M}_{\mathrm{D}, 12}$ and $\tilde{M}_{\mathrm{D}, 13}$ are zero and there are two general solutions (c.f. [32]):

$$
\tilde{M}_{\mathrm{D}, i 2}^{ \pm}=i z \sqrt{\frac{M_{s}}{2}}\left(0, \pm i e^{-i \xi} \sqrt{m_{2}}, \sqrt{m_{3}}\right), \quad \tilde{M}_{\mathrm{D}, i 3}^{ \pm}=i z^{-1} \sqrt{\frac{M_{s}}{2}}\left(0, \mp i e^{-i \xi} \sqrt{m_{2}}, \sqrt{m_{3}}\right)
$$


The solution $\tilde{M}_{\mathrm{D}}^{+}$with $\xi=\psi+\pi$ equals to $\tilde{M}_{\mathrm{D}}^{-}$with $\xi=\psi$. It allows us to consider only one solution $\tilde{M}_{D}^{+}$on the interval $0 \leq \xi<2 \pi$. In what follows we therefore omit the superscript.$+{ }^{4}$

The mixing angles of the active-sterile neutrinos are defined as follows:

$$
2 \vartheta_{\alpha}^{2} \equiv \sum_{I}\left|\left(M_{\mathrm{D}} M_{s}^{-1}\right)_{\alpha I}\right|^{2}=\sum_{I}\left|\left(V^{*} \tilde{M}_{\mathrm{D}} M_{s}^{-1}\right)_{\alpha I}\right|^{2}=\frac{1}{M_{s}^{2}} \sum_{I}\left|\left(V^{*} \tilde{M}_{\mathrm{D}}\right)_{\alpha I}\right|^{2} .
$$

Inserting the explicit solution (3.6) for $\tilde{M}_{\mathrm{D}}$ results in

$$
\vartheta_{\alpha}^{2}=\frac{|z|^{2}}{4 M_{s}}\left|\sqrt{m_{3}} V_{\alpha 3}-i e^{i \xi} \sqrt{m_{2}} V_{\alpha 2}\right|^{2}+\frac{1}{4 M_{s}|z|^{2}}\left|\sqrt{m_{3}} V_{\alpha 3}+i e^{i \xi} \sqrt{m_{2}} V_{\alpha 2}\right|^{2}
$$

For $|z| \gg 1$ the contribution of $\tilde{M}_{\mathrm{D}, i 3}$ is suppressed compared with that of $\tilde{M}_{\mathrm{D}, i 2}$ and therefore we neglect the former (we will comment below on the case $|z| \gtrsim 1$ ).

As the value of the Majorana phase $\xi$ is undetermined experimentally, the condition $\vartheta_{\alpha}=0$ is satisfied iff $m_{3}\left|V_{\alpha 3}\right|^{2}=m_{2}\left|V_{\alpha 2}\right|^{2}$ (we neglect second term on the r.h.s. of (3.8)). For the electron flavour $(\alpha=e)$ it translates into

$$
\sin ^{2} \theta_{12} \frac{m_{2}}{m_{3}}=\tan ^{2} \theta_{13},
$$

which, in principle, can be satisfied only for non-zero $\theta_{13}$. This result has been already obtained in [17].

The bounds on the parameters of the mass matrix $\mathcal{M}_{\nu}$ at the $3 \sigma$ level that we use are shown in table 1 . Note that in this paper we do not take into account statistical correlations between different oscillation parameters, allowing them to vary independently within their $3 \sigma$ intervals. Consequently, we obtain the $3 \sigma$ intervals for the combinations of parameters, entering eq. (3.9): ${ }^{5}$

$$
\begin{aligned}
0.043 & <\sin ^{2} \theta_{12} \frac{m_{2}}{m_{3}}<0.070, \\
0.010(0.014) & <\tan ^{2} \theta_{13}<0.039(0.042),
\end{aligned}
$$

They imply that the relation (3.9) does not hold exactly for the neutrino oscillation parameters, presented in table 1 . Therefore the mixing angle $\vartheta_{e}^{2}$ cannot become zero, but has a non-trivial lower bound. To find the minimal value that it can reach, we consider the ratio of the angles $\vartheta_{e}^{2} /\left(\vartheta_{e}^{2}+\vartheta_{\mu}^{2}+\vartheta_{\tau}^{2}\right)$. Due to the unitarity of $V$, the denominator is

$$
\sum_{\alpha} \vartheta_{\alpha}^{2} \approx \frac{1}{2 M_{s}^{2}} \sum_{\alpha, \beta, \gamma} V_{\alpha \beta}^{*} \tilde{M}_{\mathrm{D}, \beta 2} V_{\alpha \gamma} \tilde{M}_{\mathrm{D}, \gamma 2}^{*}=\left.\frac{1}{2 M_{s}^{2}} \sum_{\beta} \tilde{\mid} M_{\mathrm{D}, \beta 2}\right|^{2}=\frac{|z|^{2}}{4 M_{s}}\left(m_{2}+m_{3}\right) .
$$

\footnotetext{
${ }^{4}$ Unlike the parametrizations used e.g. in ref. $[17,32,33]$ this way of parametrizing the solution of the see-saw equations shows that there is only one branch of solutions, with all other related to it via redefinitions $N_{2} \leftrightarrow N_{3}$ and shift of the Majorana phases. In particular in the parametrization we used it is much easier to analyze whether mixing angles become zero. The relation $|z|=\exp (\operatorname{Im} \omega)$ holds, where the parameter $\omega$ was employed in [17].

${ }^{5}$ Throughout this paper whenever two numbers are given instead of one, the first is based on the results of the Daya Bay experiment [18], and the second one (in parentheses) is obtained based on the result of application of the RENO bounds [19] (see table 1).
} 


\begin{tabular}{|r|r|r|r|}
\hline Normal hierarchy & Inverted hierarchy & Normal hierarchy & Inverted hierarchy \\
\hline$T_{e} \leq 0.15$ & $0.02 \leq T_{e} \leq 0.98$ & $T_{e} \leq 0.17$ & $0.02 \leq T_{e} \leq 0.98$ \\
$0.09 \leq T_{\mu} \leq 0.89$ & $0 \leq T_{\mu} \leq 0.60$ & $0.07 \leq T_{\mu} \leq 0.92$ & $0 \leq T_{\mu} \leq 0.63$ \\
$0.08 \leq T_{\tau} \leq 0.88$ & $2 \times 10^{-4}\left(7 \times 10^{-5}\right) \leq T_{\tau} \leq 0.62$ & $0.06 \leq T_{\tau} \leq 0.90$ & $0 \leq T_{\tau} \leq 0.65$ \\
\hline \multicolumn{2}{|r|}{ The ranges are based on $2 \sigma$ bounds } & The ranges are based on $3 \sigma$ bounds \\
\hline
\end{tabular}

Table 2. The ratio of the sterile neutrino mixing with a given flavour $\alpha$ to the sum of the three mixings, $T_{\alpha}$ (defined by (3.12)). Left table shows the upper and lower values of $T_{\alpha}$ when parameters of neutrino oscillations are allowed to vary within their $2 \sigma$ boundaries (taken from [3]). The right table shows the results when the parameters of active neutrino oscillations are varied within their $3 \sigma$ limits (see table 1). For the explanation of the numbers in parentheses, see footnote 5 .

Let us denote the ratio of the mixing of sterile neutrinos with one flavour to the sum of all mixings by $T_{\alpha}$,

$$
T_{\alpha} \equiv \frac{\vartheta_{\alpha}^{2}}{\sum_{\beta} \vartheta_{\beta}^{2}} .
$$

Then we get the following expression for $T_{e}$ :

$$
T_{e}=\frac{\left|i e^{i \xi} c_{13} s_{12} \sqrt{\frac{m_{2}}{m_{3}}}-s_{13}\right|^{2}}{1+\frac{m_{2}}{m_{3}}} .
$$

The minimum is achieved if we push $\theta_{12}$ and $\Delta m_{21}^{2}$ to their $3 \sigma$ lower boundaries, $\theta_{13}$ and $\Delta m_{31}^{2}$ to their upper boundaries, and choose $\xi=-\pi / 2$. The maximum is achieved when we set $\Delta m_{31}^{2}$ equal to its lower bound, $\theta_{13}, \theta_{12}$ and $\Delta m_{21}^{2}$ to their upper bounds, and by choosing the Majorana phase $\alpha=\pi / 2$. The bounds on $T_{e}$ from table 2 translate into the bound for the muon and tau flavours combined:

$$
0.83 \leq T_{\mu}+T_{\tau}
$$

The minimum and maximum of different $T_{\alpha}$ are listed in the table 2 and in figure 1.

This analysis was conducted in approximation of large $|z|$. See appendix $\mathrm{C}$ for the account of finite- $|z|$ effects.

\subsection{Inverted hierarchy}

Similarly to the previous case, for the inverted hierarchy we get a solution of the see-saw equations (3.3)

$$
\tilde{M}_{\mathrm{D}, i 2}=i z \sqrt{\frac{M_{s}}{2}}\left(e^{-i \zeta} \sqrt{m_{1}}, i e^{-i \xi} \sqrt{m_{2}}, 0\right), \quad \tilde{M}_{\mathrm{D}, i 3}=i z^{-1} \sqrt{\frac{M_{s}}{2}}\left(e^{-i \zeta} \sqrt{m_{1}}, \quad-i e^{-i \xi} \sqrt{m_{2}}, 0\right)
$$

for $0 \leq \xi<2 \pi$. In this case $\vartheta_{\mu}^{2}$ or $\vartheta_{\tau}^{2}$ can become very suppressed, as we will show soon.

The mixing angles are

$$
\vartheta_{\alpha}^{2}=\frac{|z|^{2}}{4 M_{s}}\left|\sqrt{m_{1}} V_{\alpha 1}-i e^{i(\xi-\zeta)} \sqrt{m_{2}} V_{\alpha 2}\right|^{2}+\frac{1}{4 M_{s}|z|^{2}}\left|\sqrt{m_{1}} V_{\alpha 1}+i e^{i(\xi-\zeta)} \sqrt{m_{2}} V_{\alpha 2}\right|^{2}
$$


For $|z| \gg 1$ they can become close to zero only if $\sqrt{m_{1}}\left|V_{\alpha 1}\right|=\sqrt{m_{2}}\left|V_{\alpha 2}\right|$. For $\alpha=\mu$ this condition translates into

$$
\left|\tan \theta_{12}+\sin \theta_{13} \tan \theta_{23} e^{-i \phi}\right|=\sqrt{\frac{m_{2}}{m_{1}}}\left|1-\sin \theta_{13} \tan \theta_{12} \tan \theta_{23} e^{-i \phi}\right| .
$$

For the parameter set close to the best fit, left-hand side is less than the right-hand side, because then $\sin \theta_{13} \approx 0$, while $\tan \theta_{12}<1$ and $m_{1} \approx m_{2}$. To attain the equality one has to push left-hand side up and the right-hand side down. $\phi=0$ makes phases of both complex terms inside $|\ldots|$ on the left-hand side equal, thereby the absolute value of their sum becomes maximal. Simultaneously the right-hand side becomes minimal. For this specific choice of the Dirac angle the equality (3.17) turns into

$$
\frac{\sqrt{\frac{m_{2}}{m_{1}}}-\tan \theta_{12}}{\sqrt{\frac{m_{2}}{m_{1}}} \tan \theta_{12}+1}=\sin \theta_{13} \tan \theta_{23}
$$

The $3 \sigma$ bounds for inverted hierarchy in general are the same as for the normal one (see table 1) with the exception of the "atmospheric" mass difference, that slightly differs. Using these values we find

$$
0.14<\frac{\sqrt{\frac{m_{2}}{m_{1}}}-\tan \theta_{12}}{\sqrt{\frac{m_{2}}{m_{1}}} \tan \theta_{12}+1}<0.24, \quad 0.08(0.09)<\sin \theta_{13} \tan \theta_{23}<0.26
$$

We see that two regions overlap, therefore the relation (3.17) can be satisfied and $\vartheta_{\mu}^{2}$ can be zero in a wide region of values of the parameters of the neutrino oscillation matrix. See, however, section 3.4 below.

Similarly, the condition $\vartheta_{\tau}=0$ (for $\left.\phi=\pi\right)$ translates into

$$
\frac{\sqrt{\frac{m_{2}}{m_{1}}}-\tan \theta_{12}}{\sqrt{\frac{m_{2}}{m_{1}}} \tan \theta_{12}+1}=\sin \theta_{13} \cot \theta_{23}
$$

and can be satisfied, because the quantity on the right hand side varies from 0.07 (0.09) to $0.24(0.25)$, well within the range of (3.19). ${ }^{6}$

On the other hand, $\vartheta_{e}$ can be zero only if

$$
\cot \theta_{12}=\sqrt{\frac{m_{2}}{m_{1}}}
$$

can be realized. The left hand side is always larger than the right hand side (within the $3 \sigma$ region), therefore no $\vartheta_{e}$ suppression can occur. However it is important to know what

\footnotetext{
${ }^{6}$ It was pointed out in [17] that both $\vartheta_{\mu}$ and $\vartheta_{\tau}$ can be suppress in inverted hierarchy, for $\theta_{13}=0$. For this to happen the relation $\sqrt{\frac{m_{2}}{m_{1}}}=\tan \theta_{12}$ should hold, as one can also see from eqs. (3.18) and (3.20). The corresponding value of $\theta_{12}$ is however well outside the $3 \sigma$ interval. The general case $\theta_{13} \neq 0$ has not been analyzed in [17].
} 
minimal value this mixing angle can reach. According to eq. (3.16) electron mixing angle is given by

$$
\vartheta_{e}^{2}=\frac{|z|^{2}}{4 M_{s}} \cos ^{2} \theta_{13}\left(m_{1} \cos ^{2} \theta_{12}+m_{2} \sin ^{2} \theta_{12}+\sin (\xi-\zeta) \sin 2 \theta_{12} \sqrt{m_{1} m_{2}}\right) .
$$

For $\xi-\zeta=-\pi / 2$ this quantity is minimal

$$
\vartheta_{e, \text { min }}^{2}=\frac{|z|^{2}}{4 M_{s}^{2}} \cos ^{2} \theta_{13}\left(\sqrt{m_{1}} \cos \theta_{12}-\sqrt{m_{2}} \sin \theta_{12}\right)^{2} .
$$

To compare it with the other mixing angles, we note that the relation

$$
\sum_{\alpha} \vartheta_{\alpha}^{2} \approx \frac{|z|^{2}}{4 M_{s}}\left(m_{1}+m_{2}\right)
$$

holds (similar to eq. (3.11) in the case of normal hierarchy). Therefore

$$
\frac{\vartheta_{e, \min }^{2}}{\sum_{\alpha} \vartheta_{\alpha}^{2}}=\frac{\cos ^{2} \theta_{13}}{1+\frac{m_{2}}{m_{1}}}\left(\cos \theta_{12}-\sqrt{\frac{m_{2}}{m_{1}}} \sin \theta_{12}\right)^{2} .
$$

The results of the analysis are listed in table 2 and figure 1. From the upper bound on $T_{\alpha}$ we derive the bound

$$
T_{\mu}+T_{\tau} \geq 0.02
$$

We see that in this mass hierarchy it is possible for the overall coupling of the sterile neutrino to both $\mu$ and $\tau$ flavours to become tiny compared to the electron flavour coupling.

\subsection{Minimal mixing angles in the $\nu$ MSM}

Finally, we find the minimal values of the sterile neutrino mixing angles in the $\nu$ MSM, compatible with the neutrino oscillation data. These angles will turn out to be much smaller than the experimental upper bounds in all regions of masses, probed by the experiments. A general solution of the see-saw equations (3.8), (3.16) gives $\vartheta$ as a function of $|z|$ :

$$
\vartheta_{\alpha}^{2}=A_{\alpha}|z|^{2}+\frac{B_{\alpha}}{|z|^{2}}
$$

with coefficients $A_{\alpha}$ and $B_{\alpha}$ independent of $|z|$. The minimum of this expression is reached for $|z|_{\alpha}^{2}=\sqrt{B_{\alpha} / A_{\alpha}} \geq 1$ and is given by

$$
\left(\vartheta_{\alpha}^{2}\right)_{\min }=2 \sqrt{A_{\alpha} B_{\alpha}} .
$$

To find an absolute lower bound on the mixing angle for a given sterile neutrino mass, we vary this expression over the parameters of neutrino oscillations. The resulting mixing angles and the corresponding values of $|z|$ are listed in table $3 .^{7}$ One can see that the values presented therein do not depend significantly on the $3 \sigma$ upper bound on $\theta_{13}$ that we 

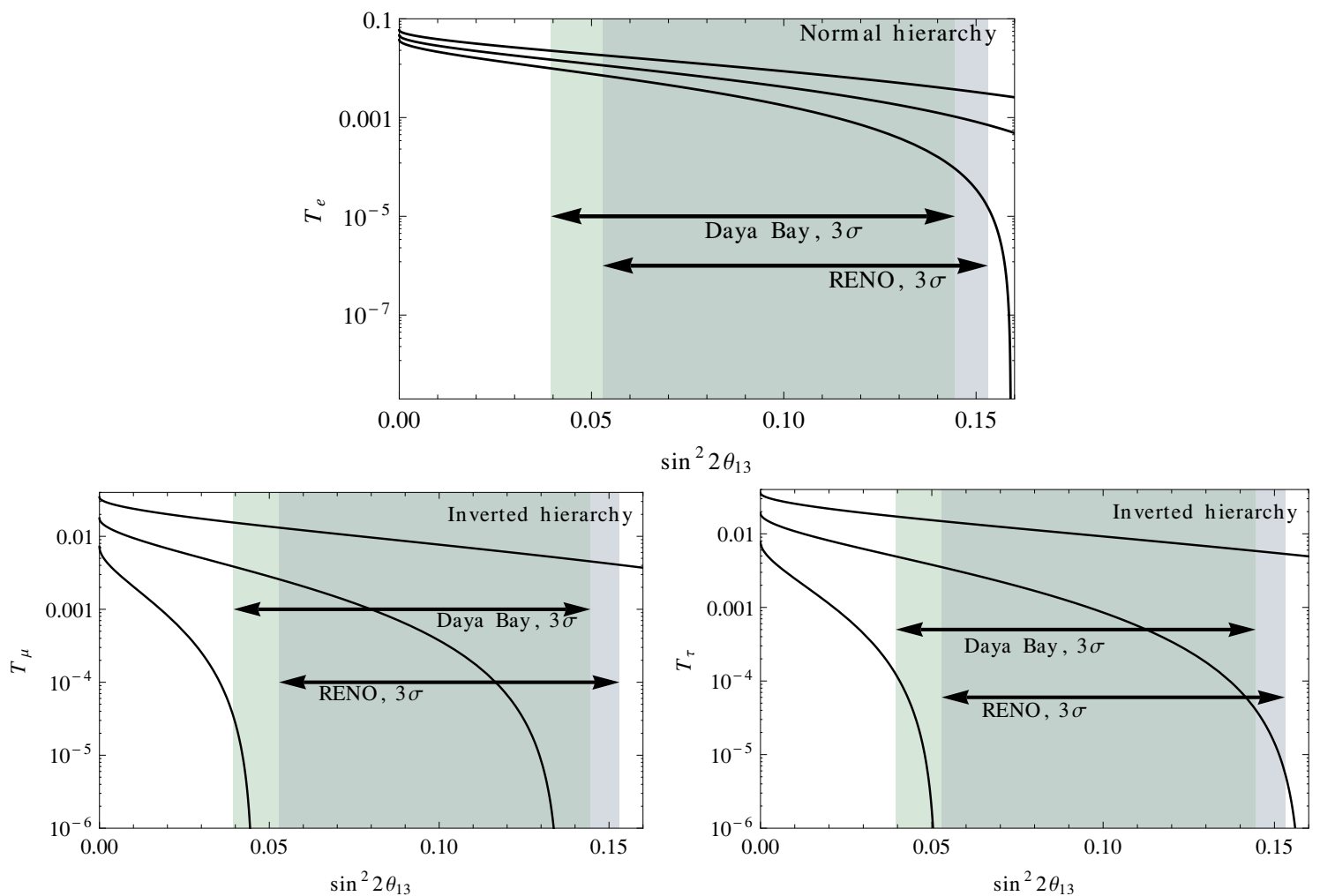

Figure 1. The minimal ratios of mixing angles $T_{\alpha}=\vartheta_{\alpha}^{2} / \sum \vartheta_{\beta}^{2}$. The upper figure depicts normal hierarchy, two lower ones - IH. In all figures, the lower curve corresponds to the choice of the mixing angles and mass splittings that minimizes the ratio within the $3 \sigma$ range, upper - that maximizes it, middle employs the best-fit parameters (for details of the choices, see sections 3.2, 3.3). CP-phases are $\xi=-\pi / 2$ for the $T_{e}$-plot, $\phi=0, \xi-\zeta=\pi / 2$ for $T_{\mu}$, and $\phi=\pi, \xi-\zeta=-\pi / 2$ for $T_{\tau}$. The bands Daya Bay and RENO correspond to the $3 \sigma$ ranges of $\theta_{13}$, indicated by the corresponding experiments $[18,19]$.

choose. The only exception is the minimum of the $\vartheta_{e}$ angle. In this case the exact value of the upper bound on $\theta_{13}$ defines how close $A_{\alpha}$, and hence $\left(\vartheta_{\alpha}^{2}\right)_{\min }$, can come to zero.

For the mixing angles $\vartheta_{\mu, \tau}^{2}$ in the case of inverted hierarchy $A_{\alpha}=0, B_{\alpha} \neq 0$ and formally for infinitely large $|z|$ they would become zero. The value of $|z|$, however, is bounded from above, $|z|<z_{\max }$, by the requirement that none of three mixing angles exceeds its upper bound (for quantitative estimates of $z_{\max }$, look at figure 9). Therefore the couplings to $\mu$ and $\tau$ neutrinos remain finite. Estimates of mixing angles can be provided for $B_{\alpha}$ given by $L_{\alpha}^{I H}$ (C.2), (C.3), along with $A_{\mu, \tau}=0, z=z_{\max }$

$$
\vartheta_{\mu, \tau}^{2} \gtrsim 2 \times 10^{-8} \frac{\mathrm{MeV}}{M_{s} z_{\max }^{2}},
$$

\footnotetext{
${ }^{7}$ Notice, that the ratio of the mixing angles $\vartheta_{\alpha}^{2} / \vartheta_{\beta}^{2}$ does not reach its minimum when (3.28) is satisfied. The values of $|z|$ for which the bounds on the lifetime are relaxed the most are those when some of the mixing angles reach their upper experimentally allowed value.
} 


\begin{tabular}{|c|c|c|}
\hline Flavour $\alpha$ & $\left(\vartheta_{\alpha}^{2}\right)_{\min } @ 1 \mathrm{MeV}$ & $|z|$ \\
\hline$e$ & $7 \times 10^{-10}$ & $2.2(2.4)$ \\
$\mu, \tau$ & $10^{-8}$ & 1.5 \\
\hline
\end{tabular}

(a) $\mathrm{NH}$, best-fit

\begin{tabular}{|c|c|c|}
\hline Flavour $\alpha$ & $\left(\vartheta_{\alpha}^{2}\right)_{\min } @ 1 \mathrm{MeV}$ & $|z|$ \\
\hline$e$ & $10^{-10}\left(4 \times 10^{-11}\right)$ & $6.2(9.8)$ \\
$\mu$ & $8 \times 10^{-10}\left(6 \times 10^{-10}\right)$ & $5.4(6.3)$ \\
$\tau$ & $1.2 \times 10^{-9}\left(1.0 \times 10^{-10}\right)$ & $4.6(5.1)$ \\
\hline
\end{tabular}

(b) $\mathrm{NH}, 3 \sigma$

\begin{tabular}{|c|c|c|}
\hline Flavour $\alpha$ & $\left(\vartheta_{\alpha}^{2}\right)_{\min } @ 1 \mathrm{MeV}$ & $|z|$ \\
\hline$e$ & $10^{-8}$ & 2.3 \\
$\mu, \tau$ & $2 \times 10^{-9}$ & 3.5 \\
\hline
\end{tabular}

(c) IH, best-fit

\begin{tabular}{|c|c|c|}
\hline Flavour $\alpha$ & $\left(\vartheta_{\alpha}^{2}\right)_{\min } @ 1 \mathrm{MeV}$ & $|z|$ \\
\hline$e$ & $6 \times 10^{-9}$ & 2.7 \\
$\mu, \tau$ & \multicolumn{2}{|c|}{ see text } \\
\hline
\end{tabular}

(d) $\mathrm{IH}, 3 \sigma$

Table 3. Minimal values of the active-sterile mixing angles $\vartheta_{\alpha}^{2}$, obtained using the best-fit values of neutrino oscillation parameters or by varying the neutrino oscillation data within their $3 \sigma$ intervals, listed in table 1 . The values for $\left(\vartheta_{\alpha}^{2}\right)_{\text {min }}$ are provided for sterile neutrinos with the mass $M_{s}=1 \mathrm{MeV}$. For other masses one should multiply them by $\left(\mathrm{MeV} / M_{s}\right)$. Columns " $|z|$ " show the values of $|z|$ for which the minimum in (3.27) is reached. For the explanation of numbers in brackets, see footnote 5 .

\section{Experimental bounds on sterile neutrino mixings}

The direct experimental searches for neutral leptons had been performed by a number of collaborations [34-46] (see e.g. [11, 12] for review of various constraints). The negative results of the searches are converted into the upper bound on $\vartheta_{\alpha} \vartheta_{\beta}$ for different flavours. If neutrino oscillations are mediated by these sterile neutrinos, these bounds can be translated into the upper bounds on parameter $|z|$ and lower bounds on sterile neutrino lifetime.

Below, we take a closer look at two main types of experiments ("peak searches" and "fixed target experiments" $)^{8}$ and describe reinterpretation of these bounds in the case, when sterile neutrinos with $\mathrm{MeV}-\mathrm{GeV}$ masses are also responsible for neutrino oscillations.

\subsection{Peak searches}

In "peak search" experiments [49-52], one considers the two-body decay of charged $\pi$ or $K$ mesons to charged lepton $\left(e^{ \pm}\right.$or $\left.\mu^{ \pm}\right)$and neutrino (see e.g. [11] for discussion). In case of the pion decay the limit on $\vartheta_{e}^{2}$ for masses in the range $60 \mathrm{MeV} \leq M_{s} \leq 130 \mathrm{MeV}$ is provided by the searches for the secondary positron peak in the decay $\pi^{+} \rightarrow e^{+} N$ to the massive sterile neutrino $N$ as compared to the primary peak coming from the $\pi^{+} \rightarrow e^{+} \nu_{e}$ decay. Recent

\footnotetext{
${ }^{8}$ The neutrinoless double-beta decay $(0 \nu \beta \beta)$ does not provide significant restrictions on the parameters of the sterile neutrinos in the type-I see-saw models (contrary to the case discussed in e.g. [11]), see discussion in $[17,47]$. In particular, this is the case in the $\nu$ MSM [48].
} 

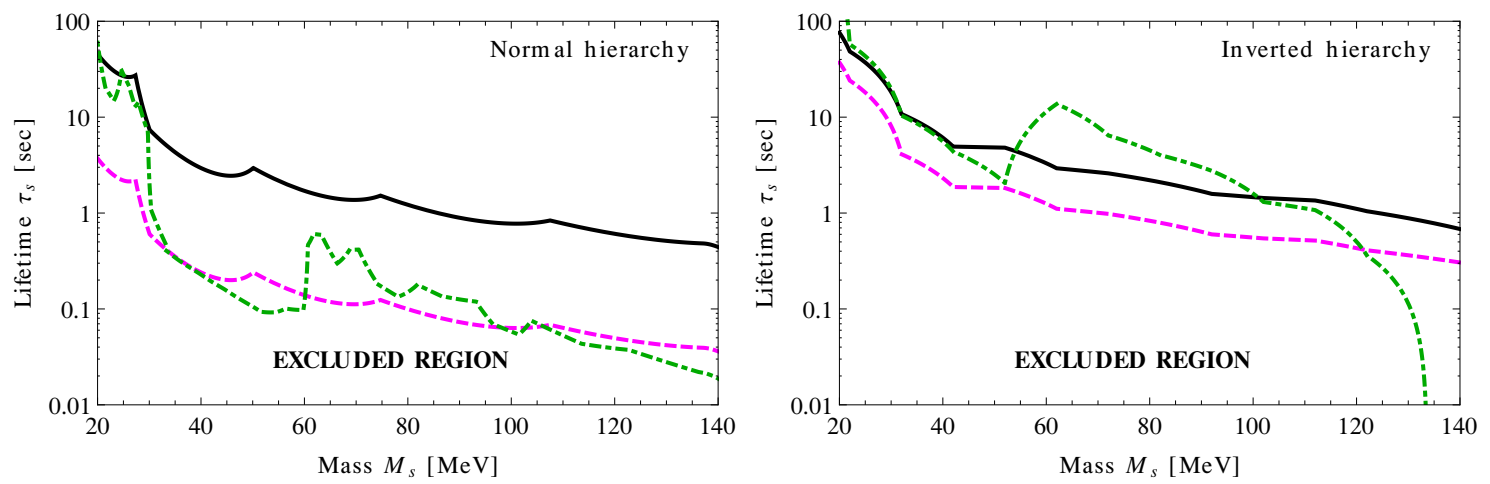

Figure 2. The lower bounds on the lifetime of sterile neutrinos, responsible for the mixings between active neutrinos of different flavours in the see-saw models (2.2). The bounds are based on the combination of negative results of direct experimental searches [34, 35, 37, 40-45] with the neutrino oscillation data [3]. The neutrino oscillation parameters are allowed to vary within their $3 \sigma$ confidence intervals to minimize the lifetime. The solid black curve is based on our reinterpretation of PS191 data only, that takes into account charged and neutral current contributions (see section 4.3). The interpretation of the PS191 experiment, taking into account only CC interactions (used e.g. in the previous works $[12,17]$ ) is shown in magenta dashed line. The bound from peak searches experiments only [37, 40-45] is plotted in green dot-dashed line.

analysis of [45] puts this limit at $\vartheta_{e}^{2}<10^{-8}$ in the mass range $60-129 \mathrm{MeV}$, for earlier results see $[36,37]$. In the smaller mass region $\left(4 \mathrm{MeV} \lesssim M_{s} \lesssim 60 \mathrm{MeV}\right)$ refs. [36, 37] provided the bound based on the change of the number of events in the primary positron peak located at energies $M_{\pi} / 2$. Similar bounds were obtained for the same mixing angle in studies of kaon decays [40] and for the $\vartheta_{\mu}^{2}$ in the decays of both pions [42-44] and kaons [40, 41].

The lower bound on the sterile neutrino lifetime $\tau_{s}$ in the model (2.2), based on the peak search data and neutrino oscillations is shown in figure 2 by dot-dashed green lines. The parameters of neutrino mixing matrix are allowed to vary within their $3 \sigma$ limits (to minimize $\tau_{s}$, while still keeping the values of all mixing angles compatible with the bounds from direct experimental searches).

\subsection{Fixed target experiments and neutral currents contribution}

The second kind of experiments ("fixed target experiments") [35, 38, 39] aims to create sterile neutrinos in decays of mesons and then searches for their decays into pairs of charged particles. Notice, that the expected signal in this second case is proportional to $\vartheta_{\alpha}^{4}$ or $\vartheta_{\alpha}^{2} \vartheta_{\beta}^{2}$ (and not to $\vartheta_{\alpha}^{2}$ as in the case of peak searches, discussed in the section 4.1). We will demonstrate below that that in the models like (2.2) (and in particular in the $\nu \mathrm{MSM}$ ) the results of some fixed target experiments should be reinterpreted and will provide stronger bounds than discussed in previous works $[11,12,17]$ (see also [53]).

\subsection{Reinterpretation of the PS191 and CHARM experiments}

The experiment PS191 at CERN was a "fixed target" type of experiment described above $[34,35]$. In searches for sterile neutrinos lighter than the pion $M_{s}<M_{\pi}$, the 

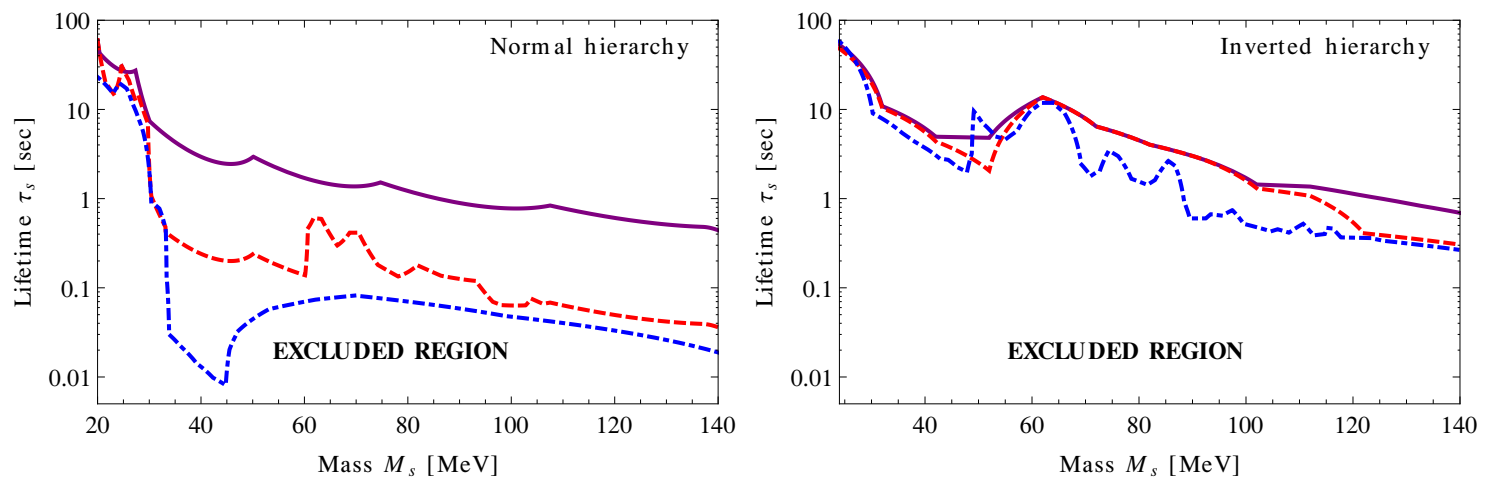

Figure 3. Comparison with the previous bounds on sterile neutrino lifetime in the $\nu$ MSM [17]. The solid purple curves represent the results of the present work, obtained by the combination of peak searches experiments [37, 40-45] together with the reanalysis of PS191, that takes into account neutral currents (a union of black and green bounds from figure 2). The red dashed curve is based on the combination of the same peak searches with the original interpretation of PS191 (i.e., with charged current interactions only). The blue dot-dashed line is taken from [17]. Notice, that the results of [17] were multiplied by a factor 2 to account for the Majorana nature of the particles (see discussion in section 4.4), that was missing therein. The difference between the red and blue lines in the case of normal hierarchy is explained by wider $3 \sigma$ intervals for neutrino oscillation data, used in [17], compared to our work.
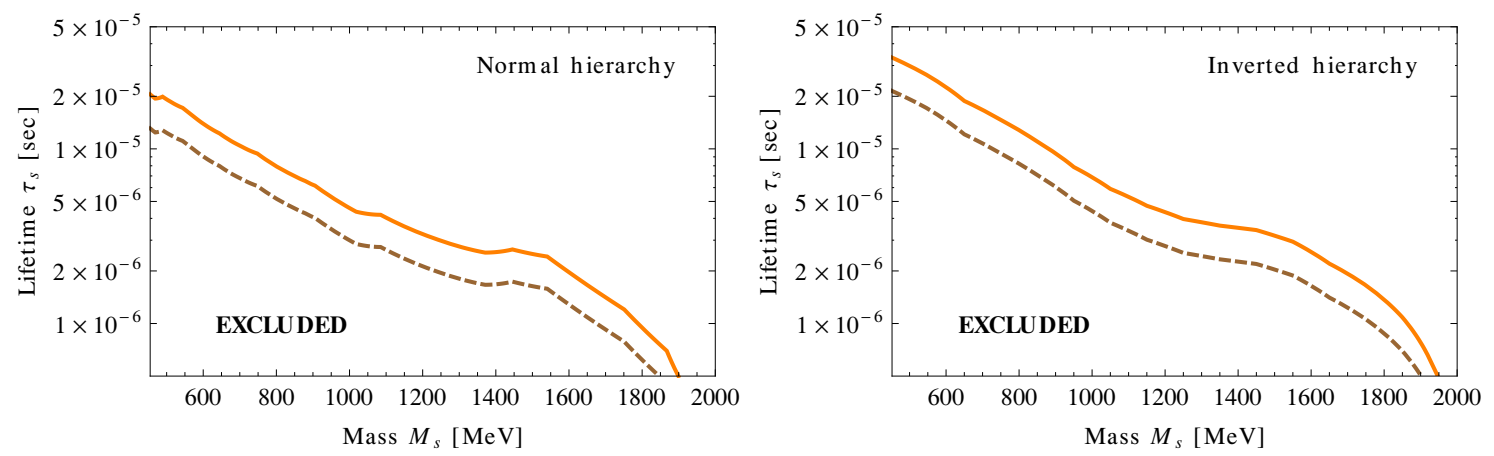

Figure 4. Comparison of the bounds on sterile neutrino lifetime (in the model (2.2)) based on the results of the CHARM experiments [39] solely (combined with the neutrino oscillation data). The orange (upper) curves correspond to the model with charged and neutral current interactions of sterile neutrinos, the brown (lower) - to the model with charged current interactions only. For details, see section 4.3 .

pair of charged particles that were searched for in the neutrino decay comprised mostly of electron and positron:

$$
\begin{aligned}
\pi^{+} / K^{+} \rightarrow e^{+}+ & N \\
& \hookrightarrow e^{+} e^{-} \nu_{\alpha},
\end{aligned}
$$

where $N$ is a sterile neutrino with the mass $M_{s}$. The first reaction in the chain is solely due to the charged-current (CC) interaction, and its rate is proportional to the $\vartheta_{e}^{2}$.

If sterile neutrinos interact through both charged and neutral currents $(\mathrm{CC}+\mathrm{NC})$ as it is the case in the models with the see-saw Lagrangian (2.2), any of three active-neutrino 
flavours may appear in the decay of $N$ in (4.1). The decay widths are [49]:

$$
\Gamma\left(N \rightarrow e^{+} e^{-} \nu_{\alpha}\right)=c_{\alpha} \vartheta_{\alpha}^{2} \frac{G_{F}^{2} M_{s}^{5}}{96 \pi^{3}},
$$

with the following definition

$$
c_{e}=\frac{1+4 \sin ^{2} \theta_{W}+8 \sin ^{4} \theta_{W}}{4}, \quad c_{\mu}=c_{\tau}=\frac{1-4 \sin ^{2} \theta_{W}+8 \sin ^{4} \theta_{W}}{4},
$$

and $\theta_{W}$ is the Weinberg's angle so that $\sin ^{2} \theta_{W} \approx 0.231$ and $c_{e} \approx 0.59, c_{\mu(\tau)} \approx 0.13$. Therefore, the total number of events inside the detector that registers electron-positron pairs would be proportional to the combination of mixing angles $\vartheta_{e}^{2} \times\left(\sum c_{\alpha} \vartheta_{\alpha}^{2}\right)$.

However, the model employed in the interpretation of the PS191 experiment [34, 35] was different, as has already been pointed in [53]. In the original analysis it was assumed that sterile neutrino interact only via charged currents, but not through neutral currents. In our language it means that $c_{e}=1, c_{\mu(\tau)}=0$ was used instead of the values (4.3). ${ }^{9}$ As was noticed above, the probability of meson decay into sterile neutrino does not alter if we exclude the neutral-current interaction, and therefore the total number of events with the electron-positron pair would be proportional to $\vartheta_{e}^{2} \times \vartheta_{e}^{2}$.

Therefore if we denote the bounds listed in $[34,35]$ as $\vartheta_{e}^{4} \leq \vartheta_{e(\exp )}^{4}$, then the bound for the $\nu$ MSM takes form

$$
\vartheta_{e}^{2}\left(\sum_{\alpha=\{e, \mu, \tau\}} c_{\alpha} \vartheta_{\alpha}^{2}\right) \leq \vartheta_{e(\exp )}^{4} .
$$

Similar bounds can be extracted from the reanalysis of meson decays into muon and sterile neutrino, that leads to replacement $e \rightarrow \mu$ in (4.4). As a result, the reinterpretation of the results of the PS191 experiment in combination with neutrino oscillation data produces up to an order of magnitude stronger bounds on lifetime than in the previous works (see figures 2 and 3 ).

Similarly, the CHARM experiment [39] provided bounds on the mixing angles of sterile neutrinos in the mass range $0.5 \mathrm{GeV} \lesssim M_{s} \lesssim 2 \mathrm{GeV}$. In the original analysis NC contributions were neglected. Therefore, to apply the results of this experiment to the case of the $\nu \mathrm{MSM}$, we reanalyzed the data as described above. In figure 4 we compare lifetime bounds coming from the CHARM experiment solely for $\mathrm{CC}$ and $\mathrm{CC}+\mathrm{NC}$ interactions of sterile neutrinos. The difference in this case is about a factor of $2{ }^{10}$

\footnotetext{
${ }^{9}$ Model described in $[34,35]$ contains only one Dirac neutrino, while in the $\nu \mathrm{MSM}$ we have two Majorana fermions. Therefore actually $c_{e}=1 / 2$ in the original model. For details see section 4.4 .

${ }^{10}$ In the case of the PS191 experiment, when using CC only for masses below the mass of pion suppression of the $\vartheta_{e}^{2}$ mixing angle due to neutrino oscillations meant that instead of $\vartheta_{e}^{2}$ bounds the lifetime is defined by the (much weaker) $\vartheta_{\mu}^{2}$ bounds. That led to the significant relaxation of the lower bound on the lifetime. If $\mathrm{NC}$ were taken into account, this was not possible anymore and therefore the lower bound on sterile neutrino lifetime became stronger by as much as the order on magnitude (black vs. magenta curve on the left panel in figure 2. In case of the CHARM experiment, both $\vartheta_{e}^{2}$ and $\vartheta_{\mu}^{2}$ are strongly constrained and switching from one constraint to another makes (numerically) much smaller difference.
} 


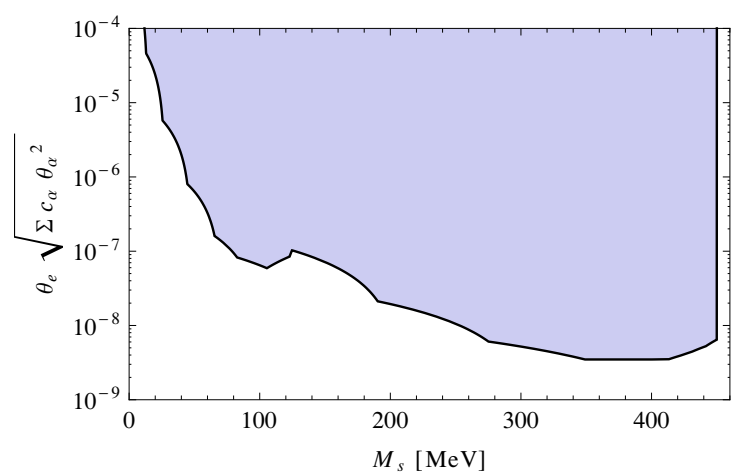

(a) Bound on the combination $\vartheta_{e} \sqrt{\sum c_{\alpha} \vartheta_{\alpha}^{2}}$

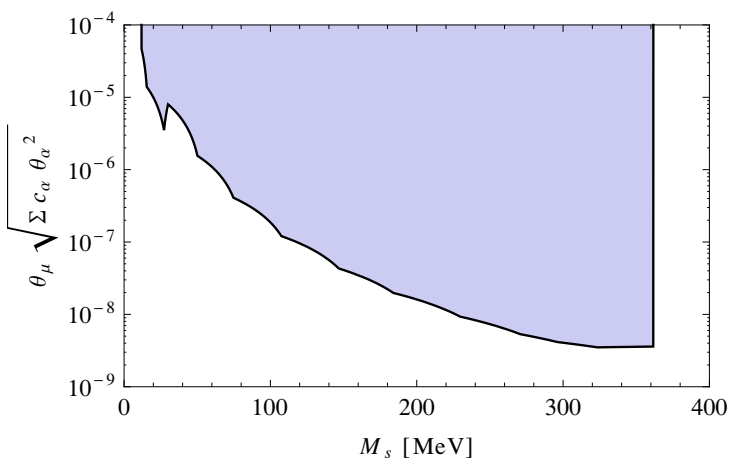

(b) Bound on the combination $\vartheta_{\mu} \sqrt{\sum c_{\alpha} \vartheta_{\alpha}^{2}}$

Figure 5. Direct accelerator bounds on the combination of active-sterile neutrino mixing angles, resulting from the reanalysis of the PS191 experiment [34, 35], taking into account decays of sterile neutrino through both charged and neutral currents and their Majorana nature. The shaded region is excluded. The case, analyzed in the original works [34, 35] (decay of sterile neutrino through the charged current only) corresponds to the choice $c_{e}=1, c_{\mu}=c_{\tau}=0$, for details, see section 4.3. We plot the bounds for two Majorana neutrinos (as in figure 6) while in the original works [34, 35] a single Dirac neutrino was analyzed.

\subsection{A note on Majorana vs Dirac neutrinos}

For completeness we briefly discuss the difference in interpreting experimental results for Majorana vs. Dirac sterile neutrinos. Similar discussion can be found e.g. in [12]. When interpreting the experimental results one should take into account that in present work we consider two Majorana sterile neutrinos, while the experimental papers often phrase their bounds in terms of the mixing with a single Dirac neutrino, that we will denote $U_{\alpha}^{2}$. In the $\nu \mathrm{MSM}$ twice more sterile neutrinos are produced per single reaction (because there are two sterile species $-N_{2}$ and $N_{3}$ ), and, owing to their Majorana nature, each sterile neutrino decays twice faster (additional charge-conjugated decay modes are present). Notice, that the mass splitting between between two sterile states $N_{2}, N_{3}$ is small $\left|M_{2}-M_{3}\right| \ll \frac{1}{2}\left(M_{2}+\right.$ $\left.M_{3}\right)=M_{s}$ and once born, the states oscillate fast into each other. Averaging over many oscillations can be accounted for by an extra factor $\frac{1}{2}$ in the number of $N_{2}$ and $N_{3}$ species. Therefore, for fixed target experiments one gets the same number of the detector events involving one Dirac sterile neutrino as one gets in the $\nu$ MSM if $\left(\vartheta_{\alpha 2}^{2}+\vartheta_{\alpha 3}^{2}\right)^{2}=U_{\alpha}^{4}$. That is, one should identify $2 \vartheta_{\alpha}^{2}$ with the measured $U_{\alpha}^{2}$ (recall (3.7) that $\left.\vartheta_{\alpha}^{2}=\frac{1}{2}\left(\vartheta_{\alpha 2}^{2}+\vartheta_{\alpha 3}^{2}\right)\right)$. In the case of peak searches, the bound $U_{\alpha}^{2}$ should be interpreted in the $\nu$ MSM as $\vartheta_{\alpha, 2}^{2}+\vartheta_{\alpha, 3}^{2} \leq U_{\alpha}^{2}$, as production of any state $N_{2}$ or $N_{3}$ contributes to the number of events in the secondary peak, i.e. again $2 \vartheta_{\alpha}^{2}$ should be identified with $U_{\alpha}^{2}$. Notice, that this factor 2 is missing in [17].

\section{Results}

In this section we summarize our results: the upper bound on the (combination of) mixing angles of sterile and active neutrinos in the see-saw models (1.2) in the range $10 \mathrm{MeV}-$ 

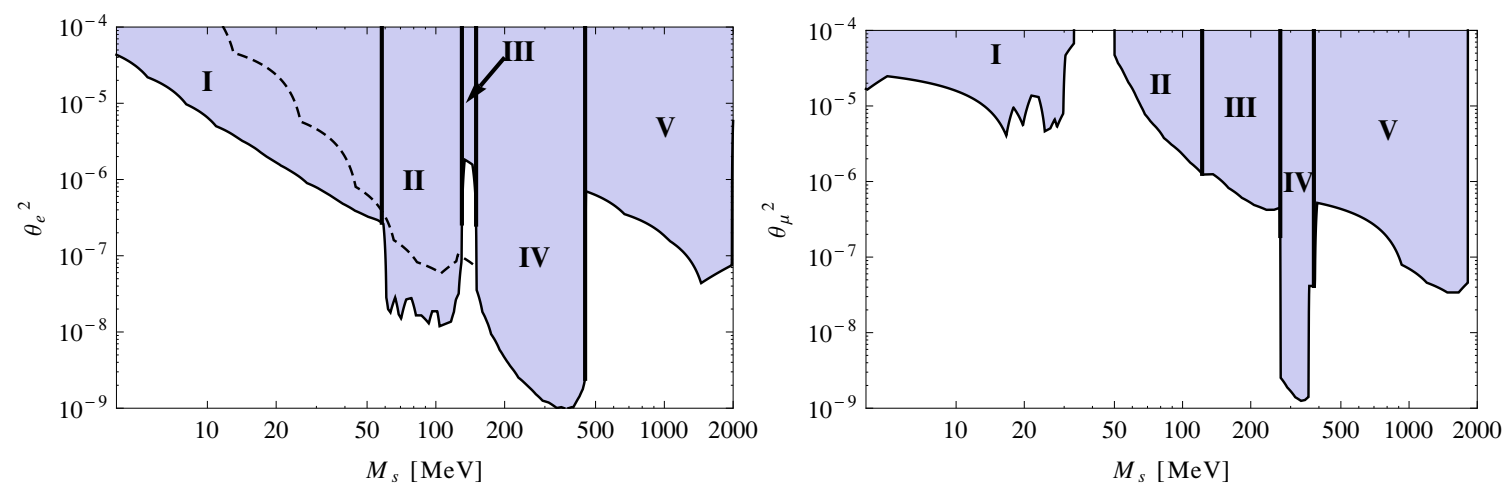

Figure 6. Direct accelerator bounds on the mixing angles. Left panel: $\vartheta_{e}^{2}$ bounds, taken from [37] (region I), [45] (region II), [40] (region III), [35, 54] (region IV) and [39] (region V). Right panel: $\vartheta_{\mu}^{2}$ bounds, taken from [42-44] (region I), [40](region II), [41] (region III), [35](region IV) and [38] (region V). The shaded regions are ruled out by the experimental findings. Dashed curve indicates mixing angle bounds given by original interpretation of PS191 experiment, but we do not use it to derive our final results, as explained in section 4.3. The bounds are shown for the Majorana neutrino and are therefore two times stronger (see section 4.4), while in the original works [34, 35] a single Dirac neutrino has been considered.

$2 \mathrm{GeV}$ and the lower bound on sterile neutrino lifetime, obtained in combination of these bounds with constraints, coming from neutrino oscillation data.

\subsection{Bounds on the mixing angles of sterile neutrinos}

For the models (2.2) (two Majorana sterile neutrinos, interacting through both charged and neutral interactions), the compilation of constraints on various combinations of activesterile mixing angles $\left(\vartheta_{e}^{2}, \vartheta_{\mu}^{2}, \vartheta_{e} \sqrt{\sum c_{\alpha} \vartheta_{\alpha}^{2}}, \vartheta_{\mu} \sqrt{\sum c_{\alpha} \vartheta_{\alpha}^{2}}\right)$ that we used in this work are plotted in figures 5 and $6 .{ }^{11}$

\subsection{The lower bound on the lifetime of sterile neutrinos}

The result of the sections 3.2-3.3, combined with these experimental bounds can be translated into the lower limits on the lifetime of sterile neutrinos. These results are presented in figures 7. Additionally, we plot the lifetime bounds for the best-fit values of the PMNS parameters yet with $\theta_{13}=0$ (as used e.g. in [12,31]). For normal hierarchy we see that our bounds with $\theta_{13} \neq 0$ are relaxed by as much as the order of magnitude at some masses, compared to $\theta_{13}=0$ case. The difference for IH is not so pronounced. Notice, that the bounds of $[12,17,31]$ were different from what we show as dashed line in figure 7 because of ignoring the neutral current contributions to the results of PS191 experiment (for details see discussion in section 4 and figures 2,3 ).

\footnotetext{
${ }^{11}$ Notice that in the published results of the PS191 experiment [35] bounds are given up to $M_{s}=400 \mathrm{MeV}$. We extend these bounds up to $450 \mathrm{MeV}$, using the PhD Thesis of J.-M. Levy [54].
} 


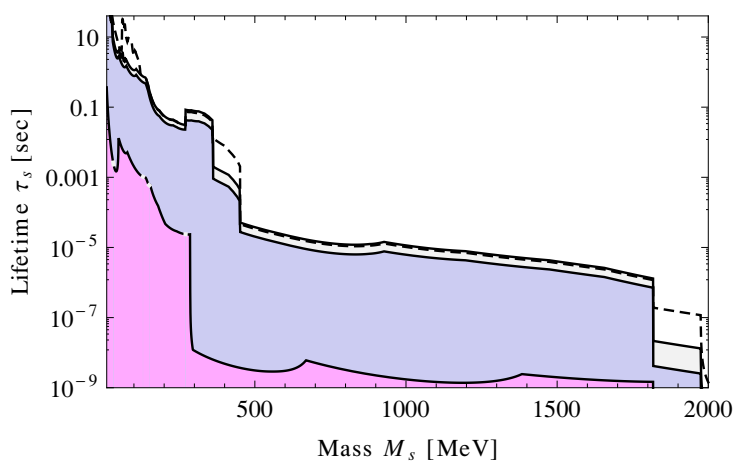

(a) Normal hierarchy, mass range $10 \mathrm{MeV}-2 \mathrm{GeV}$

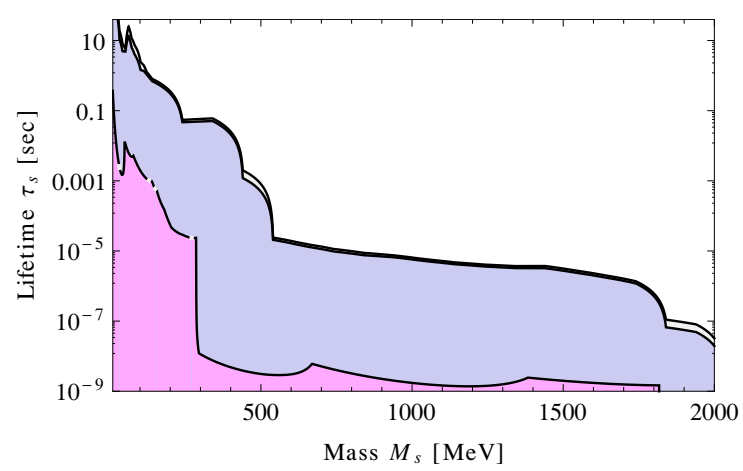

(c) Inverted hierarchy, mass range $10 \mathrm{MeV}-2 \mathrm{GeV}$

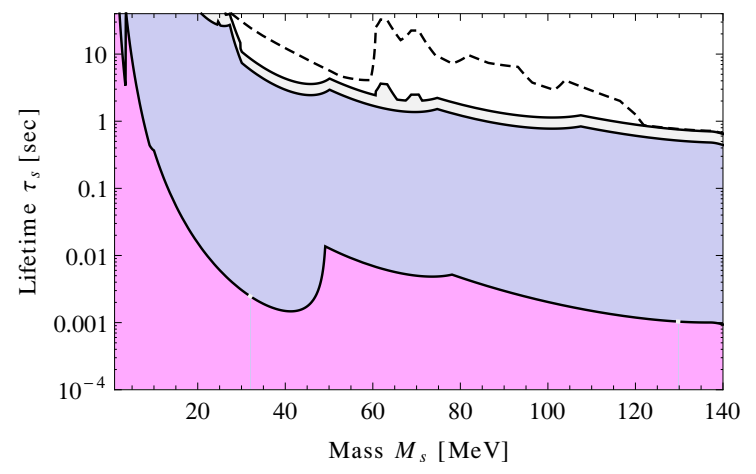

(b) Normal hierarchy, zoom at the mass range $10 \mathrm{MeV}-140 \mathrm{MeV}$

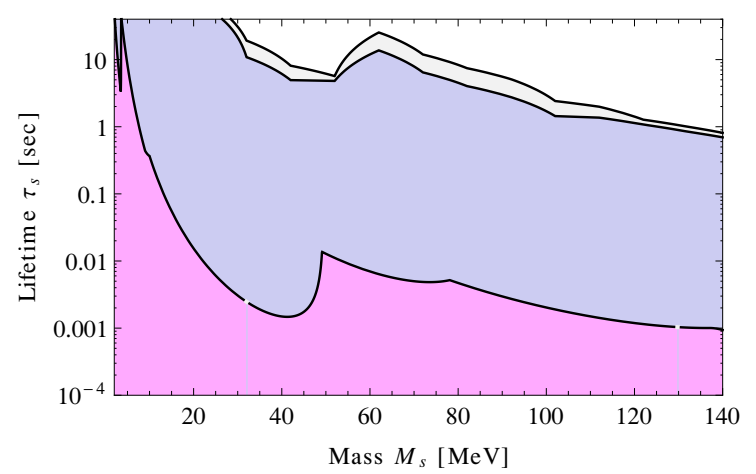

(d) Inverted hierarchy, zoom at the mass range $10 \mathrm{MeV}-140 \mathrm{MeV}$

Figure 7. The resulting lower bounds on sterile neutrino lifetime $\tau_{s}$ as a function of their mass, obtained by requiring that two Majorana sterile neutrinos are responsible for neutrino oscillations and their parameters do not contradict the negative results of direct experimental searches. In all figures the upper curve comes from using of the best fit neutrino oscillation parameters, the middle one from their variation within the $3 \sigma$ limits, and the lower one does not take into account neutrino oscillation data and puts all three mixing angles equal to their direct experimental bounds. The dashed line for $\mathrm{NH}$ corresponds to the best-fit values of PMNS parameters with $\theta_{13}=0$ and shows how much the bounds on the lifetime relax for non-zero value of $\theta_{13}$ (see text, section 5 for discussion).

\section{Discussion}

In this work we have investigated experimental restrictions on the parameters of the seesaw Lagrangian in the case when two sterile neutrinos with the masses between $\sim 10 \mathrm{MeV}$ and $2 \mathrm{GeV}$ are responsible for neutrino oscillations. Combined with the results of the direct experimental searches, the neutrino oscillation data provide stringent lower bounds on their lifetime, $\tau_{s}$ and allows to determine both maximum and minimum values of the mixing angles $\vartheta_{\alpha}^{2}$.

We have reinterpreted the results of the PS191 experiment [34, 35], following [53], by taking into account not only charged, but also neutral-current interactions (as both of these are present in the Type I see-saw Lagrangian). Our results demonstrated that below the 
mass of the pion the fixed target experiments ( $\vartheta^{4}$ experiments) provide stronger restrictions than the peak search experiments ( $\vartheta^{2}$ experiments) in case of normal hierarchy. In inverted hierarchy the reanalysis of the PS191 experiment turns out to be very important as well. In the original analysis of the CHARM experiment [38] neutral-current contributions were neglected as well and we have reinterpreted these results in a similar way to PS191. The final results are presented in figures 7 .

Future experiments (for example, NA62 in CERN, ${ }^{12}$ LBNE experiment in $\mathrm{FNAL}^{13}$ or upgraded $\mathrm{LHCb}$ experiment) have a great potential of discovering light neutral leptons of the $\nu$ MSM or significantly improving the bounds on their parameters (see discussion in [55] and [56]). Due to the strong suppression of the mixing angles $\vartheta_{e}^{2}$ in the case of NH and $\vartheta_{\mu}^{2}$ in the case of IH, the peak searches in the kaon decays (such as e.g. [57]) may miss the sterile neutrino (cf. [17]). ${ }^{14}$

Finally, sterile neutrinos with the masses in $\mathrm{MeV}-\mathrm{GeV}$ region and small mixing angles can play significant role in the early Universe (for a review see [16]). Due to their small mixing angles, the lifetime of sterile neutrinos can be long enough for them to be present in primordial plasma at $\sim \mathrm{MeV}$ temperatures, affecting the Big Bang nucleosynthesis (BBN) (for more details and references, see [14, 15, 22]). Comparison of the $3 \sigma$ lower bounds from the direct searches with the $3 \sigma$ upper bounds from BBN on the lifetime of sterile neutrinos (based on [22]) is presented in figure 8. There are no allowed values of sterile neutrinos lifetimes for $10 \mathrm{MeV} \lesssim M_{s}<140 \mathrm{MeV}$ for both types of hierarchies (i.e. the upper bound is smaller than the lower bound, see the purple double-shaded region in figure 8). This conclusion is based on the weakest BBN bound, based on the primordial Helium-4 abundance as determined from CMB observations [6, 60], which presently has large error bars. The astrophysical measurements (e.g. [61, 62]) provide a much tighter bounds on the Helium-4 abundances, further lowering the upper bound from BBN by as much as the order of magnitude (see [22] for discussion). It should be stressed that adopting the BBN bounds from the previous works $[14,15]$ would lead to essentially the same conclusion.

Our conclusion differs from that of [17], where it was argued that in the case of normal hierarchy there is an open region of parameter space compatible with both direct experimental searches and BBN bounds of [15]. This difference comes mainly from the reanalysis of the data of PS191 experiment that was carried out in the present paper (as illustrated in figure 3).

For heavier sterile neutrinos, no reliable computations of the sterile-neutrino impact on BBN were made up to the present time, and the rough estimate $\tau_{s} \lesssim 0.1 \mathrm{sec}$ is usually used instead (for more details, see [15]). This latter bound gives no substantial restriction on such heavy sterile neutrinos, since from figure 7 it can be inferred that the horizontal line $\tau_{s}=0.1 \mathrm{sec}$ is generally higher than the bounds depicted there.

Apart from their influence on BBN, the decays of sterile neutrinos may produce additional entropy and energy of plasma [22,63]. Their out-of-equilibrium behaviour may lead

\footnotetext{
${ }^{12}$ http://na62.web.cern.ch/NA62.

${ }^{13}$ http://lbne.fnal.gov/.

${ }^{14} \mathrm{GeV}$-scale sterile neutrinos in the models with extended Higgs sector [58] can be searched at the LHC [59].
} 

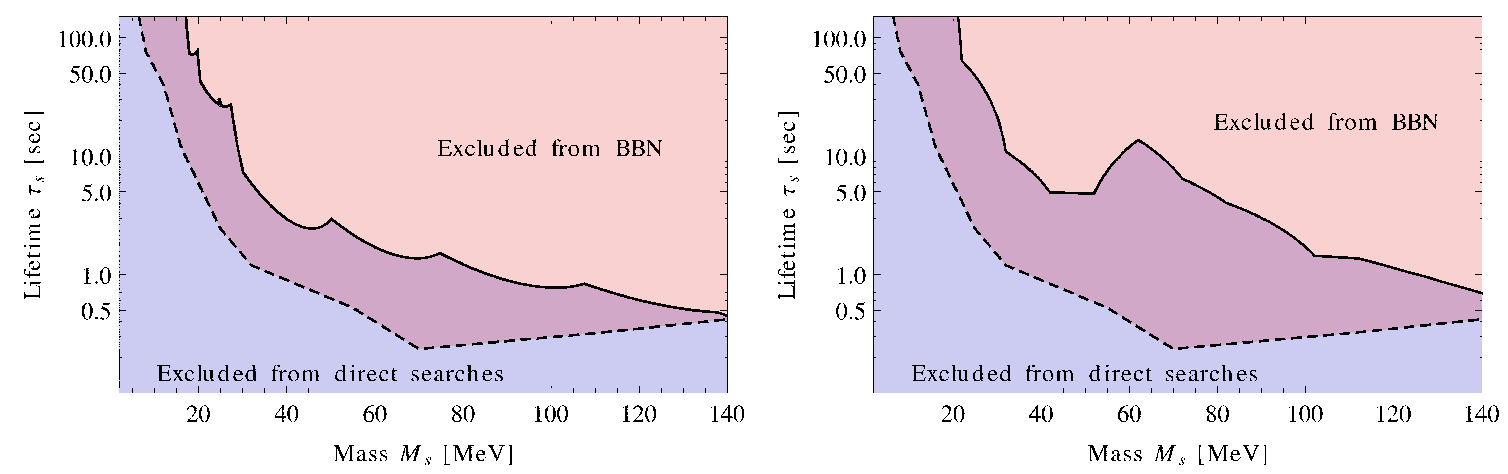

Figure 8. Experimental lower bounds on the lifetime of sterile neutrinos (solid line), combined with the upper bounds coming from the BBN (dashed line) [22]. Left panel: normal hierarchy, right panel: inverted hierarchy. Shown are $3 \sigma$ lower bounds from the figure 7 and the weakest bound from primordial nucleosynthesis (based on the CMB measurements of primordial Helium abundance, yielding $Y_{p}=0.445$ at the $3 \sigma$ level $[6,60]$, see text for details).
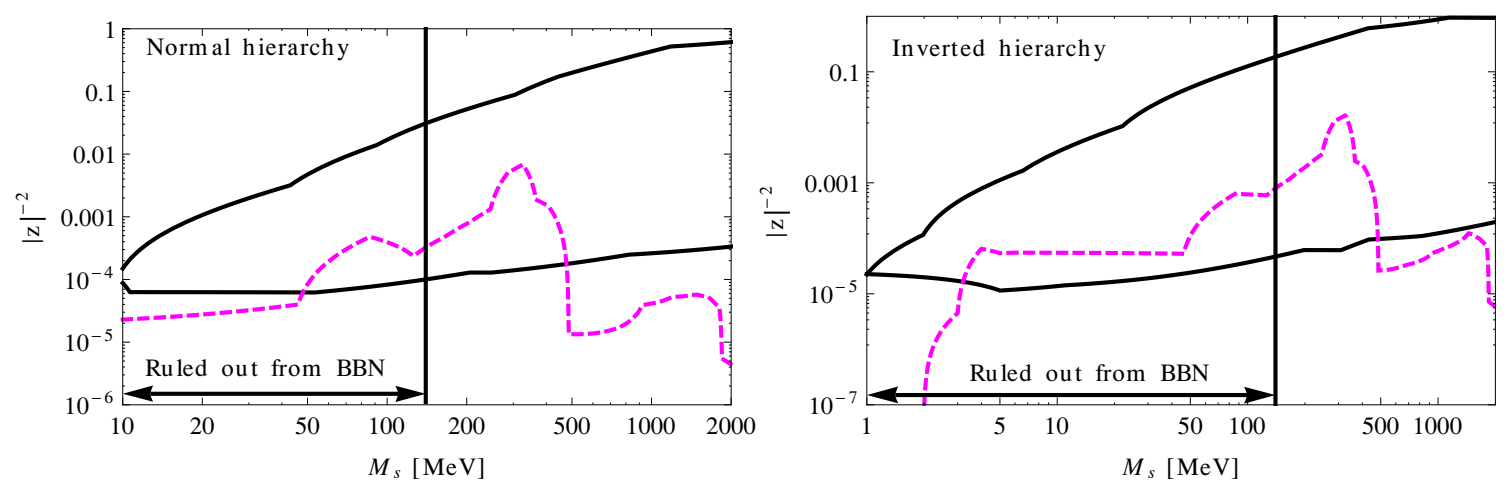

Figure 9. The region of successful baryogenesis in the $\nu$ MSM compared with the experimental upper bounds on the parameter $|z|$. The vales of $M_{s}$ and $|z|$, lying inside the black solid lines lead to the production of the observable baryon asymmetry (from [31]). The magenta dashed line marks is the lower bound on $|z|^{-2}$ (parameter, called $\epsilon$ in $[13,31]$ ) such that for smaller values at least one of the mixing angles $\vartheta_{\alpha}^{2}$ is in contradiction with direct experimental searches (for the best-fit values of the PMNS mixing angles and masses). The value of $|z|$ corresponding to the bound is what we refer to as $z_{\max }$ in section 3.4. The region to the left of $M_{s}=140 \mathrm{MeV}$ is ruled out from comparison with primordial nucleosynthesis bounds (figure 8).

as well to the successful baryogenesis scenario [29, 31, 64]; the generation of large lepton asymmetry at temperatures below the sphaleron freeze-out [13]. In figure 9 we superimpose the bounds on $|z|$, coming from the direct experimental searches on the region of parameters $\left(|z|, M_{s}\right)$ in which the successful baryogenesis is possible (the region inside the black contours marked "BAU" based on the ref. [31]). Additionally, the lepton asymmetry may be generated at $T \lesssim$ few $\mathrm{GeV}$ [13] and can affect production of dark matter sterile neutrinos [65, 66]. Sterile neutrinos with the mass of about $200 \mathrm{MeV}$ and mixing angles $\sim 10^{-7}-10^{-8}$ can affect the physics of supernova explosions [67]. Finally, our constraints may affect the low-reheating temperature cosmological scenario (see e.g. [68, 69]). 


\section{Acknowledgments}

We would like to thank T. Asaka, F. Bezrukov, A. Boyarsky, S. Eijima, D. Gorbunov, H. Ishida, S. Pascoli, T. Schwetz-Mangold, D. Semikoz, M. Shaposhnikov and J. Valle for discussion and useful comments. A.I. is also grateful to S. Vilchynskiy, Scientific and Educational Centre of the Bogolyubov Institute for Theoretical Physics in Kiev, Ukraine ${ }^{15}$ and to Ukrainian Virtual Roentgen and Gamma-Ray Observatory VIRGO.UA. ${ }^{16}$ The work of A.I. was supported in part from the Swiss-Ukrainian cooperation project (SCOPES) No. IZ73Z0_128040 of Swiss National Science Foundation. A.I. acknowledges support from the ERC Advanced Grant 20080109304.

\section{A Sterile neutrino lifetime}

For any phenomenologically interesting mass of the sterile neutrino it can decay to three neutrinos $N \rightarrow \nu \bar{\nu} \nu$ and it results in contribution

$$
B_{\nu \nu \nu}^{e}=B_{\nu \nu \nu}^{\mu}=B_{\nu \nu \nu}^{\tau}=1
$$

to the total sum in eq. (1.2) over decay products $X$.

If $M_{s}>2 M_{e} \approx 1.0 \mathrm{MeV}$, then a new decay channel appears: $N \rightarrow e^{+} e^{-} \nu$. It corresponds to

$$
\begin{aligned}
B_{e e \nu}^{\alpha}=\left(\frac{1}{4} \pm \sin ^{2} \theta_{W}+2 \sin ^{4} \theta_{W}\right)\left[\left(1-14 S_{e}^{2}-2 S_{e}^{4}-12 S_{e}^{6}\right) \sqrt{1-4 S_{e}^{2}}\right. \\
\left.+12 S_{e}^{4}\left(S_{e}^{4}-1\right) \ln \left(\frac{1-3 S_{e}^{2}-\left(1-S_{e}^{2}\right) \sqrt{1-4 S_{e}^{2}}}{S_{e}^{2}\left(1+\sqrt{1-4 S_{e}^{2}}\right)}\right)\right] \\
+2 \sin ^{2} \theta_{W}\left(2 \sin ^{2} \theta_{W} \pm 1\right)\left[S_{e}^{2}\left(2+10 S_{e}^{2}-12 S_{e}^{4}\right) \sqrt{1-4 S_{e}^{2}}\right. \\
\left.+6 S_{e}^{4}\left(1-2 S_{e}^{2}+2 S_{e}^{4}\right) \ln \left(\frac{1-3 S_{e}^{2}-\left(1-S_{e}^{2}\right) \sqrt{1-4 S_{e}^{2}}}{S_{e}^{2}\left(1+\sqrt{1-4 S_{e}^{2}}\right)}\right)\right]
\end{aligned}
$$

where $S_{X}=M_{X} / M_{s}, \theta_{W}$ is the Weinberg angle, and sign "+" corresponds to $\alpha=e$ while "" in the other case. For $S_{\mu}<\frac{1}{2}$ we should add up to a sum terms $B_{\mu \mu \nu}^{\alpha}$ that can be obtained from $B_{e e \nu}^{\alpha}$ if one changes $S_{e} \rightarrow S_{\mu}$ and if sign "+" corresponds to $\alpha=\mu$, "-" - to $\alpha=e, \tau$.

Other $B$-coefficients are

$$
\begin{aligned}
B_{e \mu \nu}^{e, \mu} & =1-8 S_{\mu}^{2}+8 S_{\mu}^{6}-S_{\mu}^{8}-24 S_{\mu}^{4} \ln S_{\mu}, \quad B_{e \mu}^{\tau}=0, \quad\left(S_{\mu}+S_{e}<1\right), \\
B_{\pi \nu}^{\alpha} & =6 \pi^{2} \frac{f_{\pi}^{2}}{M_{s}^{2}}\left(1-S_{\pi}^{2}\right)^{2}, \quad\left(S_{\pi}<1, M_{\pi} \approx 140 \mathrm{MeV}, f_{\pi} \approx 130 \mathrm{MeV}\right), \\
B_{\eta \nu}^{\alpha} & =6 \pi^{2} \frac{f_{\eta}^{2}}{M_{s}^{2}}\left(1-S_{\eta}^{2}\right)^{2}, \quad\left(S_{\eta}<1, M_{\eta} \approx 550 \mathrm{MeV}, f_{\eta} \approx 155 \mathrm{MeV}\right),
\end{aligned}
$$

\footnotetext{
${ }^{15}$ http://sec.bitp.kiev.ua/.

${ }^{16}$ http://virgo.org.ua/.
} 


$$
\begin{aligned}
& B_{\pi e}^{e}=12 \pi^{2} \frac{f_{\pi}^{2}}{M_{s}^{2}} V_{u d}^{2}\left(\left(1-S_{e}^{2}\right)^{2}-S_{\pi}^{2}\left(1+S_{e}^{2}\right)\right) \sqrt{\left(1-\left(S_{\pi}-S_{e}\right)^{2}\right)\left(1-\left(S_{\pi}+S_{e}\right)^{2}\right)}, \quad\left(S_{e}+S_{\pi}<1\right), \\
& B_{\pi \mu}^{\mu}=12 \pi^{2} \frac{f_{\pi}^{2}}{M_{s}^{2}} V_{u d}^{2}\left(\left(1-S_{\mu}^{2}\right)^{2}-S_{\pi}^{2}\left(1+S_{\mu}^{2}\right)\right) \sqrt{\left(1-\left(S_{\pi}-S_{\mu}\right)^{2}\right)\left(1-\left(S_{\pi}+S_{\mu}\right)^{2}\right)}, \quad\left(S_{\mu}+S_{\pi}<1\right) .
\end{aligned}
$$

In the last expression quantity $V_{u d} \approx 0.97$ is the element of the Cabibbo-KobayashiMaskawa (CKM) quark matrix. Expression for $B_{K e}^{e}$, that is non-zero for mass range $S_{e}+S_{K}<1\left(M_{K} \approx 495 \mathrm{MeV}\right)$, can be derived from $B_{\pi e}^{e}$ by simultaneous change $S_{\pi} \rightarrow$ $S_{K}, V_{u d} \rightarrow V_{u s} \approx 0.23, f_{\pi} \rightarrow f_{K} \approx 160 \mathrm{MeV}$. Moreover, if we change in the resulting expression $S_{e}$ by $S_{\mu}$, we get $B_{K \mu}^{\mu}$ (for $S_{\mu}+S_{K}<1$ ). What concerns B-coefficients with $\alpha=\tau$ and $X=(\pi e, \pi \mu, K e, K \mu)$, they are all zero. For masses of sterile neutrino, that are larger than the mass of the $\rho$-meson $M_{\rho} \approx 780 \mathrm{MeV}$, other $B_{X}$ appear. We do not list list them in the present paper, however they can be read from [12].

\section{B PMNS parametrization}

In section 3 we use the standard definition of the PMNS matrix (cf. e.g. [1]):

$$
\begin{aligned}
V & =\left(\begin{array}{ccc}
1 & 0 & 0 \\
0 & c_{23} & s_{23} \\
0 & -s_{23} & c_{23}
\end{array}\right)\left(\begin{array}{ccc}
c_{13} & 0 & s_{13} \\
0 & e^{i \phi} & 0 \\
-s_{13} & 0 & c_{13}
\end{array}\right)\left(\begin{array}{ccc}
c_{12} & s_{12} & 0 \\
-s_{12} & c_{12} & 0 \\
0 & 0 & 1
\end{array}\right) \\
& =\left(\begin{array}{ccc}
c_{12} c_{13} & c_{13} s_{12} & s_{13} \\
-c_{23} s_{12} e^{i \phi}-c_{12} s_{13} s_{23} & c_{12} c_{23} e^{i \phi}-s_{12} s_{13} s_{23} & c_{13} s_{23} \\
s_{23} s_{12} e^{i \phi}-c_{12} c_{23} s_{13} & -c_{12} s_{23} e^{i \phi}-c_{23} s_{12} s_{13} & c_{13} c_{23}
\end{array}\right) .
\end{aligned}
$$

where $c_{i j}=\cos \theta_{i j}$, and $s_{i j}=\sin \theta_{i j}$. The active neutrino mixing matrix is given by expression (3.1).

\section{Ratio of sterile neutrino mixing angles for $|z| \sim 1$}

As the expressions (3.8) and (3.16) show, the mixing angles have two terms: one is proportional to $|z|^{2}$ and another to $|z|^{-2}$ (recall that $|z| \geq 1$ ). It was shown in section 3.3 that for inverted hierarchy the $|z|^{2}$-term can be zero for $\vartheta_{\mu}^{2}$ and $\vartheta_{\tau}^{2}$, while the $|z|^{-2}$ term in general stays finite.

For a given value of $|z|$, the $|z|^{-2}$-term is bounded from above. According to (3.8) and (3.16), its maximum is realized simultaneously with the maximal value of

$$
L_{\alpha}^{N H}=\left|V_{\alpha 3}+i e^{i \xi} \sqrt{\frac{m_{2}}{m_{3}}} V_{\alpha 2}\right|^{2}
$$

in the normal hierarchy, and

$$
L_{\alpha}^{I H}=\left|V_{\alpha 1}+i e^{i(\xi-\zeta)} \sqrt{\frac{m_{2}}{m_{1}}} V_{\alpha 2}\right|^{2}
$$

in the inverted hierarchy. 
Analysis, similar to that of the sections $3.2-3.3$ shows that

$$
L_{e}^{N H} \leq 0.2, \quad L_{\mu}^{N H} \leq 1.1, \quad L_{\tau}^{N H} \leq 1.1, \quad L_{e}^{I H} \leq 1.96, \quad L_{\mu}^{I H} \leq 1.3, \quad L_{\tau}^{I H} \leq 1.3 .
$$

These bounds allow to estimate the contribution of the $|z|^{-2}$-terms to the whole sum of the squared mixing angles

$$
\sum_{\alpha} \vartheta_{\alpha}^{2}=\frac{m_{1}+m_{2}+m_{3}}{4 M_{s}}\left(|z|^{2}+\frac{1}{|z|^{2}}\right) .
$$

The ratio of (C.1)-(C.2) to (C.4) gives

$$
R_{\alpha}^{N H}=\frac{L_{\alpha}^{N H}}{\left(1+\frac{m_{2}}{m_{3}}\right)} \frac{1}{|z|^{4}+1}, \quad R_{\alpha}^{I H}=\frac{L_{\alpha}^{I H}}{\left(1+\frac{m_{2}}{m_{1}}\right)} \frac{1}{|z|^{4}+1} .
$$

For $z \sim 1$ it can become of order unity. However, we restrict ourselves to the sufficiently large values $z \gtrsim 10$, that are consistent with the upper bound, indicated by the experiments (see figure 9)

$$
R_{e}^{N H} \lesssim 2 \times 10^{-5}, \quad R_{\mu, \tau}^{N H} \lesssim 10^{-4}, \quad R_{e}^{I H} \lesssim 10^{-4}, \quad R_{\mu, \tau}^{I H} \lesssim 5 \times 10^{-5} .
$$

Comparison these results with the lower bounds (table 2) we see that $z^{-1}$ terms are unimportant for the for all mixing angles in $\mathrm{NH}$ and $\vartheta_{e}$ in $\mathrm{IH}$. What concerns the remaining angles $\vartheta_{\mu}$ and $\vartheta_{\tau}$ in IH, they can be substantially modified by account of $z^{-1}$-terms, but anyway each of them can become small enough, compared to the other angles, as explained in next section. As a corollary, analysis and results of sections 3.2, 3.3 do not change significantly for large enough values of $z$.

Open Access. This article is distributed under the terms of the Creative Commons Attribution License which permits any use, distribution and reproduction in any medium, provided the original author(s) and source are credited.

\section{References}

[1] A. Strumia and F. Vissani, Neutrino masses and mixings and..., hep-ph/0606054 [INSPIRE].

[2] T. Schwetz, M. Tortola and J.W. Valle, Three-flavour neutrino oscillation update, New J. Phys. 10 (2008) 113011 [arXiv:0808.2016] [INSPIRE].

[3] T. Schwetz, M. Tortola and J. Valle, Where we are on $\theta_{13}$ : addendum to 'global neutrino data and recent reactor fluxes: status of three-flavour oscillation parameters', New J. Phys. 13 (2011) 109401 [arXiv:1108.1376] [InSPIRE].

[4] G. Fogli, E. Lisi, A. Marrone, A. Palazzo and A. Rotunno, Evidence of $\theta_{13}>0$ from global neutrino data analysis, Phys. Rev. D 84 (2011) 053007 [arXiv: 1106.6028] [INSPIRE].

[5] Particle Data Group collaboration, K. Nakamura et al., Review of particle physics, J. Phys. G 37 (2010) 075021 [inSPIRE].

[6] WMAP collaboration, E. Komatsu et al., Seven-year Wilkinson Microwave Anisotropy Probe (WMAP) observations: cosmological interpretation, Astrophys. J. Suppl. 192 (2011) 18 [arXiv:1001.4538] [INSPIRE]. 
[7] P. Minkowski, $\mu \rightarrow$ er at a rate of one out of 1-billion muon decays?, Phys. Lett. B 67 (1977) 421 [INSPIRE].

[8] P. Ramond, The family group in Grand Unified Theories, hep-ph/9809459 [INSPIRE].

[9] R.N. Mohapatra and G. Senjanović, Neutrino mass and spontaneous parity violation, Phys. Rev. Lett. 44 (1980) 912 [InSPIRE].

[10] T. Yanagida, Horizontal symmetry and masses of neutrinos, Prog. Theor. Phys. 64 (1980) 1103 [INSPIRE].

[11] A. Atre, T. Han, S. Pascoli and B. Zhang, The search for heavy Majorana neutrinos, JHEP 05 (2009) 030 [arXiv:0901.3589] [INSPIRE].

[12] D. Gorbunov and M. Shaposhnikov, How to find neutral leptons of the $\nu M S M$ ?, JHEP 10 (2007) 015 [arXiv:0705.1729] [INSPIRE].

[13] M. Shaposhnikov, The $\nu M S M$, leptonic asymmetries and properties of singlet fermions, JHEP 08 (2008) 008 [arXiv:0804.4542] [INSPIRE].

[14] A. Dolgov, S. Hansen, G. Raffelt and D. Semikoz, Cosmological and astrophysical bounds on a heavy sterile neutrino and the KARMEN anomaly, Nucl. Phys. B 580 (2000) 331 [hep-ph/0002223] [INSPIRE].

[15] A. Dolgov, S. Hansen, G. Raffelt and D. Semikoz, Heavy sterile neutrinos: bounds from big bang nucleosynthesis and SN1987A, Nucl. Phys. B 590 (2000) 562 [hep-ph/0008138] [INSPIRE].

[16] A. Boyarsky, O. Ruchayskiy and M. Shaposhnikov, The role of sterile neutrinos in cosmology and astrophysics, Ann. Rev. Nucl. Part. Sci. 59 (2009) 191 [arXiv:0901.0011] [INSPIRE].

[17] T. Asaka, S. Eijima and H. Ishida, Mixing of active and sterile neutrinos, JHEP 04 (2011) 011 [arXiv:1101.1382] [INSPIRE].

[18] DAYA-BAY collaboration, F. An et al., Observation of electron-antineutrino disappearance at Daya Bay, Phys. Rev. Lett. 108 (2012) 171803 [arXiv:1203.1669] [INSPIRE].

[19] RENO collaboration, J. Ahn et al., Observation of reactor electron antineutrino disappearance in the RENO experiment, Phys. Rev. Lett. 108 (2012) 191802 [arXiv:1204.0626] [INSPIRE].

[20] MINOS collaboration, P. Adamson et al., Improved search for muon-neutrino to electron-neutrino oscillations in MINOS, Phys. Rev. Lett. 107 (2011) 181802 [arXiv:1108.0015] [INSPIRE].

[21] T2K collaboration, K. Abe et al., Indication of electron neutrino appearance from an accelerator-produced off-axis muon neutrino beam, Phys. Rev. Lett. 107 (2011) 041801 [arXiv:1106.2822] [INSPIRE].

[22] O. Ruchayskiy and A. Ivashko, Restrictions on the lifetime of sterile neutrinos from primordial nucleosynthesis, arXiv:1202.2841 [INSPIRE].

[23] V. Gorkavenko and S. Vilchynskiy, Some constraints on the Yukawa parameters in the neutrino modification of the Standard Model ( $\nu M S M)$ and CP-violation, Eur. Phys. J. C 70 (2010) 1091 [arXiv:0907.4484] [INSPIRE].

[24] J. Orloff et al., International conference on the seesaw mechanism, World Scientific, Singapore (2005). 
[25] J. Schechter and J. Valle, Neutrino masses in $\mathrm{SU}(2) \times \mathrm{U}(1)$ theories, Phys. Rev. D 22 (1980) 2227 [INSPIRE].

[26] J. Schechter and J. Valle, Neutrino decay and spontaneous violation of lepton number, Phys. Rev. D 25 (1982) 774 [inSPIRE].

[27] W. Rodejohann and J. Valle, Symmetrical parametrizations of the lepton mixing matrix, Phys. Rev. D 84 (2011) 073011 [arXiv:1108.3484] [INSPIRE].

[28] T. Asaka, S. Blanchet and M. Shaposhnikov, The $\nu M S M$, dark matter and neutrino masses, Phys. Lett. B 631 (2005) 151 [hep-ph/0503065] [INSPIRE].

[29] T. Asaka and M. Shaposhnikov, The $\nu M S M$, dark matter and baryon asymmetry of the universe, Phys. Lett. B 620 (2005) 17 [hep-ph/0505013] [INSPIRE].

[30] A. Boyarsky, A. Neronov, O. Ruchayskiy and M. Shaposhnikov, The masses of active neutrinos in the $\nu$ MSM from X-ray astronomy, JETP Lett. 83 (2006) 133 [hep-ph/0601098] [INSPIRE].

[31] L. Canetti and M. Shaposhnikov, Baryon asymmetry of the universe in the $\nu M S M$, JCAP 09 (2010) 001 [arXiv: 1006.0133] [INSPIRE].

[32] M. Shaposhnikov, A possible symmetry of the $\nu$ MSM, Nucl. Phys. B 763 (2007) 49 [hep-ph/0605047] [INSPIRE].

[33] J. Casas and A. Ibarra, Oscillating neutrinos and $\mu \rightarrow e \gamma$, Nucl. Phys. B 618 (2001) 171 [hep-ph/0103065] [INSPIRE].

[34] G. Bernardi et al., Search for neutrino decay, Phys. Lett. B 166 (1986) 479 [InSPIRE].

[35] G. Bernardi et al., Further limits on heavy neutrino couplings, Phys. Lett. B 203 (1988) 332 [INSPIRE].

[36] D. Britton et al., Measurement of the $\pi^{+} \rightarrow e^{+}$neutrino branching ratio, Phys. Rev. Lett. 68 (1992) 3000 [INSPIRE].

[37] D. Britton et al., Improved search for massive neutrinos in $\pi^{+} \rightarrow e^{+}$neutrino decay, Phys. Rev. D 46 (1992) 885 [InSPIRE].

[38] $\nu$ TeV and E815 collaborations, A. Vaitaitis et al., Search for neutral heavy leptons in a high-energy neutrino beam, Phys. Rev. Lett. 83 (1999) 4943 [hep-ex/9908011] [INSPIRE].

[39] CHARM collaboration, F. Bergsma et al., A search for decays of heavy neutrinos in the mass range 0.5-2.8 GeV, Phys. Lett. B 166 (1986) 473 [INSPIRE].

[40] T. Yamazaki et al., Search for heavy neutrinos in kaon decay, in Proceedings of high energy physics conference, volume 1, Leipzig Germany (1984), pg. 262 [INSPIRE].

[41] R. Hayano et al., Heavy neutrino search using $K_{\mu 2}$ decay, Phys. Rev. Lett. 49 (1982) 1305 [INSPIRE].

[42] D. Bryman and T. Numao, Search for massive neutrinos in $\pi^{+} \rightarrow \mu^{+}$neutrino decay, Phys. Rev. D 53 (1996) 558 [INSPIRE].

[43] R. Abela et al., Search for an admixture of heavy neutrino in pion decay, Phys. Lett. B 105 (1981) 263 [Erratum ibid. B 106 (1981) 513] [INSPIRE].

[44] M. Daum et al., Search for admixtures of massive neutrinos in the decay $\pi^{+} \rightarrow \mu^{+}$neutrino, Phys. Rev. D 36 (1987) 2624 [INSPIRE]. 
[45] PIENU collaboration, M. Aoki et al., Search for massive neutrinos in the decay $\pi \rightarrow e \nu$, Phys. Rev. D 84 (2011) 052002 [arXiv:1106.4055] [INSPIRE].

[46] DELPHI collaboration, P. Abreu et al., Search for neutral heavy leptons produced in Z decays, Z. Phys. C 74 (1997) 57 [Erratum ibid. C 75 (1997) 580] [INSPIRE].

[47] M. Blennow, E. Fernandez-Martinez, J. Lopez-Pavon and J. Menendez, Neutrinoless double beta decay in seesaw models, JHEP 07 (2010) 096 [arXiv: 1005.3240] [INSPIRE].

[48] F. Bezrukov, $\nu M S M$-predictions for neutrinoless double beta decay, Phys. Rev. D 72 (2005) 071303 [hep-ph/0505247] [INSPIRE].

[49] R. Shrock, New tests for and bounds on, neutrino masses and lepton mixing, Phys. Lett. B 96 (1980) 159 [INSPIRE].

[50] R.E. Shrock, General theory of weak leptonic and semileptonic decays. 1. Leptonic pseudoscalar meson decays, with associated tests for and bounds on, neutrino masses and lepton mixing, Phys. Rev. D 24 (1981) 1232 [INSPIRE].

[51] R.E. Shrock, General theory of weak processes involving neutrinos. 2. Pure leptonic decays, Phys. Rev. D 24 (1981) 1275 [INSPIRE].

[52] R.E. Shrock, Pure leptonic decays with massive neutrinos and arbitrary Lorentz structure, Phys. Lett. B 112 (1982) 382 [INSPIRE].

[53] A. Kusenko, S. Pascoli and D. Semikoz, New bounds on MeV sterile neutrinos based on the accelerator and Super-Kamiokande results, JHEP 11 (2005) 028 [hep-ph/0405198] [INSPIRE].

[54] J.-M. Levy, Production et désintégration de neutrinos massifs (in French), doctoral thesis, University of Paris, Paris France (1986).

[55] LBNE collaboration, T. Akiri et al., The 2010 interim report of the long-baseline neutrino experiment collaboration physics working groups, arXiv:1110.6249 [INSPIRE].

[56] K. Abazajian et al., Light sterile neutrinos: a white paper, arXiv:1204.5379 [INSPIRE].

[57] A. Shaykhiev, Y. Kudenko and A. Khotyantsev, Searches for heavy neutrinos in the decays of positively charged kaons, Phys. Atom. Nucl. 74 (2011) 788 [Yad. Fiz. 74 (2011) 814] [INSPIRE].

[58] A. Kusenko, Sterile neutrinos, dark matter and the pulsar velocities in models with a Higgs singlet, Phys. Rev. Lett. 97 (2006) 241301 [hep-ph/0609081] [INSPIRE].

[59] I.M. Shoemaker, K. Petraki and A. Kusenko, Collider signatures of sterile neutrinos in models with a gauge-singlet Higgs, JHEP 09 (2010) 060 [arXiv: 1006.5458] [INSPIRE].

[60] J. Dunkley et al., The Atacama Cosmology Telescope: cosmological parameters from the 2008 power spectra, Astrophys. J. 739 (2011) 52 [arXiv: 1009.0866] [INSPIRE].

[61] E. Aver, K.A. Olive and E.D. Skillman, An MCMC determination of the primordial helium abundance, JCAP 04 (2012) 004 [arXiv: 1112.3713] [INSPIRE].

[62] Y. Izotov and T. Thuan, The primordial abundance of ${ }^{4} H e$ : evidence for non-standard big bang nucleosynthesis, Astrophys. J. 710 (2010) L67 [arXiv:1001.4440] [INSPIRE].

[63] G.M. Fuller, C.T. Kishimoto and A. Kusenko, Heavy sterile neutrinos, entropy and relativistic energy production and the relic neutrino background, arXiv:1110.6479 [INSPIRE]. 
[64] E.K. Akhmedov, V. Rubakov and A.Y. Smirnov, Baryogenesis via neutrino oscillations, Phys. Rev. Lett. 81 (1998) 1359 [hep-ph/9803255] [INSPIRE].

[65] X.-D. Shi and G.M. Fuller, A new dark matter candidate: nonthermal sterile neutrinos, Phys. Rev. Lett. 82 (1999) 2832 [astro-ph/9810076] [INSPIRE].

[66] M. Laine and M. Shaposhnikov, Sterile neutrino dark matter as a consequence of $\nu M S M-i n d u c e d$ lepton asymmetry, JCAP 06 (2008) 031 [arXiv:0804.4543] [INSPIRE].

[67] G.M. Fuller, A. Kusenko and K. Petraki, Heavy sterile neutrinos and supernova explosions, Phys. Lett. B 670 (2009) 281 [arXiv:0806.4273] [INSPIRE].

[68] G. Gelmini, S. Palomares-Ruiz and S. Pascoli, Low reheating temperature and the visible sterile neutrino, Phys. Rev. Lett. 93 (2004) 081302 [astro-ph/0403323] [INSPIRE].

[69] G. Gelmini, E. Osoba, S. Palomares-Ruiz and S. Pascoli, MeV sterile neutrinos in low reheating temperature cosmological scenarios, JCAP 10 (2008) 029 [arXiv:0803.2735] [INSPIRE]. 